\title{
LOCAL DIRICHLET FORMS, HODGE THEORY, AND THE NAVIER-STOKES EQUATIONS ON TOPOLOGICALLY ONE-DIMENSIONAL FRACTALS
}

\author{
MICHAEL HINZ AND ALEXANDER TEPLYAEV
}

\begin{abstract}
We consider finite energy and $L^{2}$ differential forms associated with strongly local regular Dirichlet forms on compact connected topologically onedimensional spaces. We introduce notions of local exactness and local harmonicity and prove the Hodge decomposition, which in our context says that the orthogonal complement to the space of all exact 1-forms coincides with the closed span of all locally harmonic 1-forms. Then we introduce a related Hodge Laplacian and define a notion of harmonicity for finite energy 1-forms. As a corollary, under a certain capacity-separation assumption, we prove that the space of harmonic 1 -forms is nontrivial if and only if the classical Cech cohomology is nontrivial. In the examples of classical self-similar fractals these spaces typically are either trivial or infinitely dimensional. Finally, we study Navier-Stokes type models and prove that under our assumptions they have only steady state divergence free solutions. In particular, we solve the existence and uniqueness problem for the Navier-Stokes and Euler equations for a large class of fractals that are topologically one-dimensional but can have arbitrary Hausdorff and spectral dimensions.
\end{abstract}

\section{Contents}

1. Introduction

2. Setup and preliminaries

3. Locally constant functions and Dirichlet subdomains

4. Locally exact and locally harmonic 1-forms

5. Nontriviality of the first Cech cohomology

6. Form Laplacian and harmonic 1-forms

7. Navier-Stokes equations on fractals

8. The case of resistance forms

Acknowledgements

References

Received by the editors May 30, 2013.

2010 Mathematics Subject Classification. Primary 31E05, 60J45; Secondary 28A80, 31C25, 35J25, 35Q30, 46L87, 47F05, 58J65, 60J60, 81Q35.

The research of the first author was supported in part by the Alexander von Humboldt Foundation (Feodor Lynen Research Fellowship Program) and carried out during a stay at the Department of Mathematics, University of Connecticut, Storrs, Connecticut 06269-3009.

The research of the first and second authors was supported in part by NSF grant DMS-0505622. 


\section{INTRODUCTION}

In this article we study the finite energy de Rham complex on compact connected topologically one-dimensional spaces $X$ which carry a strongly local regular Dirichlet (energy) form $(\mathcal{E}, \mathcal{F})$. There are large classes of fractals of arbitrary Hausdorff dimension $1 \leq d_{H}<\infty$ and various possible spectral dimensions that have these properties (see 2, and the references therein; 15, 45, 48, 51, 59, 62, 63, 68, and Remark 1.1). During recent years Dirichlet forms have proven to be efficient tools in various areas of analysis and probability. Some recent papers related to our research that use Dirichlet form methods are for instance [6, 8, 9]. We investigate the de Rham complex consisting of finite energy 0 -forms and square integrable 1forms that results from constructions introduced in [19, 20, 40]. We introduce new localization techniques and prove that locally exact forms are dense in the space of 1-forms, which allows us to define a Hodge Laplacian on 1-forms.

One of the aims of our article is to generalize results previously obtained in 18 . and [40], which connect 1-forms and topology (thus we relate differential geometry on fractals and topology). These papers contain, among several other results, Hodge type decompositions for the Sierpinski gasket with its standard Dirichlet form, [18], and more generally, for finitely ramified fractals carrying a resistance form, 40. It was shown in [40, in the finitely ramified case, that the Hilbert space of harmonic forms is trivial if and only if the space is a tree. Moreover, harmonic forms 'count' the cycles of these topologically one-dimensional spaces. In contrast to classical smooth examples (e.g. [11, 41, 69]), the space of harmonic forms on self-similar fractals will often be either trivial or infinite-dimensional.

Being a Hilbert space, the space of 1-forms is self-dual, which allows us to identify 1 -forms and vector fields. Doing so allows us to introduce some elements of vector analysis, as was recently done in 33. (which generalizes earlier approaches to vector analysis on fractals; see [47, 49, 55, 64, 68]). This is a part of a more comprehensive program addressing vector equations on general, possibly fractal, spaces which carry a diffusion, a topic that is still brand new. Some related scalar PDE are discussed in [33] and first applications to magnetic fields are considered in [34,35].

In this paper we introduce hydrodynamical models on fractals, such as NavierStokes type systems (see e.g. [1,16, 65] for background). In a sense, this justifies that our notion of finite energy differential forms is mathematically natural and implies physically relevant properties of classical differential equations on fractals. Note, however, that on classical fractals (e.g. the Sierpinski gasket) there are no continuous tangent vector fields in the classical sense (see 67. for explanations), and so our notions correspond to the so-called measurable Riemannian geometry (a term introduced by Kigami in [49]).

Overall our results revolve around the the notions of local exactness and local harmonicity of differential 1-forms. As parts of the paper are quite technical, we take a moment to outline some ideas, definitions and main results. Consider a smooth compact one-dimensional manifold $M$ without boundary. Of course there is not much variety, as any such $M$ must be diffeomorphic to the circle. The de Rham complex of $M$ has the structure

$$
0 \rightarrow \Omega^{0}(M) \stackrel{d_{0}}{\rightarrow} \Omega^{1}(M) \stackrel{d_{1}}{\longrightarrow} 0,
$$

where $\Omega^{0}(M)=C^{\infty}(M)$ and $\Omega^{1}(M)$ are the spaces of smooth functions and 1forms on $M$, respectively, and $d_{i}$ denote the respective instances of the exterior 
derivative. If, although not needed to study de Rham theory, the manifold $M$ is endowed with a Riemannian structure, then we can consider the Dirichlet integral

$$
\mathcal{E}_{M}(f)=\int_{M}|d f|^{2} \text { dvol, } \quad f \in C^{\infty}(M),
$$

where $d=d_{0}$ and dvol denotes the Riemannian volume. Taking its closure yields a symmetric local regular Dirichlet $\left(\mathcal{E}_{M}, H^{1}(M)\right)$ form on $L_{2}(M, \mathrm{dvol})$, where $H^{1}(M)$ is the corresponding Sobolev space of functions on $M$. In the present paper we investigate general topologically one-dimensional compact metric spaces $X$ that carry a finite Radon measure $m$ with full support and a symmetric strongly local regular Dirichlet form $(\mathcal{E}, \mathcal{F})$ on $L_{2}(X, m)$. We assume that it admits a spectral gap and, to exclude potential theoretic complications, that the associated transition kernels are absolutely continuous with respect to the reference measure $m$. We will frequently make use of the related energy measures $\Gamma(f)$, which can be defined for any energy finite function $f \in \mathcal{F}$ and in particular satisfy

$$
\mathcal{E}(f)=\int_{X} d \Gamma(f)
$$

More details can be found in Section 2 or in [15, 23, 44]. For the Dirichlet form (2) for instance we observe $\Gamma(f)=|d f|^{2}$ dvol, $f \in H^{1}(M)$.

Concerning differential forms on $X$, the first question would be how to define them. We follow the approach of Cipriani and Sauvageot [19,20] that, in some sense, reverses the order of definition of the objects in (11) and (2). There the authors start with a Dirichlet form and construct a Hilbert space $\mathcal{H}$ as the closure of tensor products of bounded, energy finite functions (in a certain anti-symmetrizing norm, which may be expressed using the energy measures induced by the Dirichlet form). They refer to it as the space of 1-forms on $X$, and so will we. The space of $(m$-a.e.) bounded energy finite functions $\mathcal{B}:=\mathcal{F} \cap L_{\infty}(X, m)$ forms an algebra. A related derivation operator $\partial: \mathcal{B} \rightarrow \mathcal{H}$ takes such functions into 1 -forms, and it may be extended to an unbounded closed linear operator on $L_{2}(X, m)$ with domain $\mathcal{F}$. A special case of this construction yields Weaver's derivation for $L_{\infty}$-diffusions, [70]. In cases where both theories coexist, $\partial f$ coincides with the upper gradient of $f$ in the sense of Cheeger; see [14] and also [19, 28, 29, 53, 70. The norm in $\mathcal{H}$ is such that for 1-forms of type $g \partial f$ we have

$$
\|g \partial f\|_{\mathcal{H}}^{2}=\int_{X} g^{2} d \Gamma(f)
$$

and the collection of such 1 -forms $g \partial f$ is dense in $\mathcal{H}$. This approach is an $L_{2^{-}}$ formulation, and in general differential 1-forms $\omega \in \mathcal{H}$ cannot be evaluated pointwise. Note that for the Dirichlet form as in (2) we have in particular

$$
\|g \partial f\|_{\mathcal{H}}^{2}=\int|g d f|^{2} \text { dvol, } f, g \in C^{\infty}(M),
$$

extending (2). Therefore $\mathcal{H}$ is a generalization of the space $L_{2}\left(M, \operatorname{dvol}, \Lambda^{1} T M\right)$ of square integrable differential 1-forms on $M$, and the operator $\partial$ generalizes the closure of $d=d_{0}: C^{\infty}(M) \rightarrow L_{2}\left(M, \mathrm{dvol}, \Lambda^{1} T M\right)$ in $L_{2}(M, \mathrm{dvol})$. If in (1) we replace the spaces $\Omega^{i}(M)$ by the spaces of square integrable functions and 1-forms and consider $L_{2}$-derivations $\partial_{i}$ instead of $d_{i}$, we get an $L_{2}$-differential complex. Its generalization in our setup should read

$$
0 \rightarrow L_{2}(X, m) \stackrel{\partial_{0}}{\longrightarrow} \mathcal{H} \stackrel{\partial_{1}}{\longrightarrow} 0
$$


with $\partial_{0}=\partial$, which is an unbounded closed densely defined linear operator on $L_{2}(X, m)$ with domain $\mathcal{F}$. However, a concept of 2 -forms and a derivation $\partial_{1}$ taking 1-forms into 2 -forms is not yet fully established, and therefore writing (3) still involves the assumption that the space of 2 -forms is trivial. Wherever we state definitions motivated by this assumption we will mention it explicitly and comment on it.

A key property of the de Rham complex (in fact, of any differential complex) is $d^{2}=0$, i.e. all exact 1 -forms $\omega=d f$ are closed, $d \omega=0$. In the one-dimensional case of course all 1-forms are closed. On the other hand, every closed 1-form in the de Rham sense is locally exact by a special case of the Poincaré lemma. In [18] it has been shown that this implication cannot be expected to hold in our context. More precisely, the authors have constructed a nonlocally exact 1-form on the Sierpinski gasket, which is topologically one-dimensional and fits into our framework. Here we define local exactness as follows (cf. Definition 4.1): A 1-form $\omega \in \mathcal{H}$ is called locally exact if there exist a finite open cover $\mathcal{U}=\left\{U_{\alpha}\right\}_{\alpha \in J}$ of $X$ and functions $f_{\alpha} \in \mathcal{B}, \alpha \in J$, such that

$$
\omega \mathbf{1}_{U_{\alpha}}=\partial f_{\alpha} \mathbf{1}_{U_{\alpha}}, \alpha \in J .
$$

Then one of our main results, Theorem 4.2 (i), reads as follows.

Theorem. The collection of locally exact 1 -forms is dense in $\mathcal{H}$.

This is a strong indication that we should assume the space of 2 -forms to be trivial, as in (3): any useful definition of $\partial_{1}$ should be local. Therefore $\partial_{1}$ should vanish on locally exact forms, i.e. they should be closed. As they are dense in $\mathcal{H}$ it is not too far fetched to expect $\mathcal{H}=$ ker $\partial_{1}$.

Next, recall that in the classical one-dimensional case we have the $L_{2}$-Hodge decomposition

$$
L_{2}\left(M, \operatorname{dvol}, \Lambda^{1} T M\right)=\operatorname{Im} \partial_{0} \oplus \mathbb{H},
$$

where $I m \partial_{0}$ is the image of $\partial_{0}$ and $\mathbb{H}$ is the space of $\omega \in L_{2}\left(M, \operatorname{dvol}, \Lambda^{1} T M\right)$ that satisfy $0=\partial_{1} \omega=\partial_{0}^{*} \omega$. Here $\partial_{0}^{*}$ denotes the 0 -codifferential of $\partial_{0}$, that is, the formal adjoint of $\partial_{0}$. The space $\mathbb{H}$ is called the space of harmonic 1-forms. See for instance [24. Also in our setup Im $\partial$ is a closed subspace of $\mathcal{H}$, and therefore

$$
\mathcal{H}=\operatorname{Im} \partial \oplus \mathcal{H}^{1}(X),
$$

where $\mathcal{H}^{1}(X)=(\operatorname{Im} \partial)^{\perp}$ is the orthogonal complement of $\operatorname{Im} \partial$. For the example (2) we clearly have $\mathcal{H}^{1}(M)=\mathbb{H}$. We may ask about the significance of the space $\mathcal{H}^{1}(X)$ in the general case.

In classical theory central results of de Rham theory tell that the first de Rham cohomology

$$
H_{d R}^{1}(M):=\operatorname{ker} d_{1} / \operatorname{Im} d_{0}
$$

of a smooth compact manifold $M$ is finite-dimensional and isomorphic to the first Cech cohomology $\check{H}^{1}(M)$ of $M$; see e.g. [11,41, 69]. Moreover, the Hodge theorem tells that in every cohomology class $[\omega] \in H_{d R}^{1}(M)$ there exists a unique member of $\mathbb{H}$. Also $\mathbb{H}$ is finite-dimensional, as follows from compactness and ellipticity. In [40] it was shown that for finitely ramified fractals $X$ carrying a resistance form, the space $\mathcal{H}^{1}(X)$ is trivial if and only if $X$ is a tree. Moreover, its elements were seen to 'count' the cycles of graph approximations of these topologically one-dimensional spaces. For p.c.f. self-similar fractals for instance the space $\mathcal{H}^{1}(X)$ is therefore typically either trivial or infinite-dimensional. For combinatorially finite metric 
graphs it may be finite-dimensional, [40, and trivial if and only if the graph is a tree. Note that our theory is very different from the smooth situation, because it allows complicated gluing of circles. From a topological point of view the classical Sierpinski gasket for instance arises as a limit of spaces obtained by gluing circles with 'contradicting' orientations.

Recall that in the $L_{2}$-context cohomology groups should generally not be expected to allow simple statements on topology, as their dimensions are not topological invariants. What we prove here in our setup is the following qualitative result: If $\mathcal{H}^{1}(X)$ is nontrivial, then also the first Cech cohomology $\check{H}^{1}(X)$ of $X$ must be nontrivial, and under some potential theoretic condition a nontrivial first cohomology $\check{H}^{1}(X)$ also guarantees the nontriviality of $\mathcal{H}^{1}(X)$. To establish this result we introduce a notion of local harmonicity: A 1-form $\omega \in \mathcal{H}$ is called locally harmonic if there exist a finite open cover $\mathcal{U}=\left\{U_{\alpha}\right\}_{\alpha \in J}$ of $X$ and functions $h_{\alpha} \in \mathcal{B}, \alpha \in J$, such that each $h_{\alpha}$ is harmonic on $U_{\alpha}$ and

$$
\omega \mathbf{1}_{U_{\alpha}}=\partial h_{\alpha} \mathbf{1}_{U_{\alpha}}, \alpha \in J .
$$

Here the term 'harmonic function' is used in the Dirichlet form sense; see Section 2 , Another denseness result is Theorem 4.2 (ii).

Theorem. The collection of locally harmonic 1-forms is dense in $\mathcal{H}^{1}(X)$.

Finally, we define the notion of harmonicity for 1-forms. To do so, we introduce a Hodge Laplacian $\Delta_{1}$ on 1-forms by setting

$$
\Delta_{1}:=\partial \partial^{*}
$$

and observing the following; cf. Theorem 6.1 .

Theorem. The Hodge Laplacian $\Delta_{1}$ defines a self-adjoint operator on $\mathcal{H}$.

Of course definition (5) is made with the expectation that the space of 2-forms will be trivial, i.e. that any 1 -form on $X$ will be closed. In view of our previous results this seems most reasonable. We agree to say that a 1 -form $\omega \in \mathcal{H}$ is harmonic if it is in the kernel of $\Delta_{1}$. We characterize harmonicity in Theorem 6.2. Combined with (44) it leads to an analog of the Hodge decomposition theorem and tells us that $\mathcal{H}^{1}(X)$ should be called the space of harmonic 1-forms.

Theorem. A 1-form $\omega \in \mathcal{H}$ is harmonic if and only if $\omega \in \mathcal{H}^{1}(X)$. Consequently, a 1-form is harmonic if and only if it is orthogonal to the space of exact 1-forms, and every 1-form may be written as the orthogonal sum of an exact and a harmonic part.

As an application of our results we study Navier-Stokes type models on compact connected topologically one-dimensional spaces. On the circle the Navier-Stokes system simplifies to an Euler type equation, which has only steady state solutions. We observe a similar behavior in the present case. The main difference is that on a fractal space, one can have infinitely many nontrivial solutions corresponding to various cycles in the space. If the system is considered without boundary conditions, nontrivial solutions exist if and only if the first Čech cohomology does not vanish. If time-independent boundary conditions are imposed, there may exist additional nontrivial solutions that are gradients of harmonic functions. Note that we do not have to consider so-called tamed Navier-Stokes equations (see 60] and the references therein). 
Remark 1.1. In several places our arguments crucially rely on topological onedimensionality and compactness. However, no further structure principles are used and therefore the results apply to classical smooth examples (the real line, intervals and circles), quantum graphs (see [10] and references therein), as well as to fractals such as p.c.f. self-similar sets or nested fractals, [2, 27, 40, 45, 48, generalized Sierpinski carpets 4, 5, 57 of topological dimension one, Barlow-Evans-Laakso spaces and their generalizations [7, 42,61,63, diamond fractals [52] and some random fractals 25, 26. Fractal examples are not required to be finitely ramified 68 or self-similar. Our research is influenced by the analysis with respect to the energy measures on singular spaces [36, 39, 46, 49,. We are especially interested in applications to analysis and geometry on metric measure spaces; see [14, and the references therein; 28, 29, 53, 70.

Note in particular that there are spaces of any Hausdorff dimension $1 \leq d_{H}<\infty$ to which our results apply, for instance, spaces of Barlow-Evans-Laakso type. Of special importance is the relation between our work and [57, where the authors consider generalized Sierpinski carpets of positive two-dimensional Lebesgue measure that are topologically one-dimensional. This relation will be the subject of further study.

All this also implies that we can find examples for a broad range of spectral dimensions $d_{S}$. Recall that by definition $d_{S}=2 d_{H} / d_{w}$, where $d_{w} \geq 2$ is the socalled walk index (walk dimension). In [3] a related result for weighted graphs states that for any pair of numbers $\left(\alpha, d_{w}\right)$ with $\alpha \geq 1$ and $2 \leq d_{w} \leq 1+\alpha$, there exist a weighted graph carrying an Ahlfors $\alpha$-regular measure and a random walk with walk index $d_{w}$. For prefractal graphs $d_{w}>2$ is typical.

This paper is organized as follows. In Section 2 we start with a symmetric strongly local regular Dirichlet form $(\mathcal{E}, \mathcal{F})$ and recall the definition of energy measures, capacities and harmonic functions. We state a maximum principle and finally facts about hitting kernels. Section 3 is rather technical. It introduces Dirichlet subdomains obtained by completion from functions that are locally constant on a neighborhood of a given compact set. Using suitable covers, partitions of unity and the regularity of the Dirichlet form, the original Dirichlet domain $\mathcal{F}$ is recovered as the sum of two such subdomains for disjoint compact sets; see Theorem 3.1. Later on these results are used to study locally exact and locally harmonic 1-forms. Following [19,20,40] we introduce 1-forms and derivations in Section 4 We define and discuss the notions of local exactness and local harmonicity as outlined above. Note that in [18] and [40] the spaces under consideration possess rigid structures which support rather transparent proofs by graph approximations. In our paper we do not assume any specific cell structure, and turn instead to finite open covers in order to connect 1-forms and topology. Using solely the definition of 1-forms, it therefore seems rather difficult to describe the space $\mathcal{H}^{1}(X)$, but the notion of local harmonicity allows us to link simple 1 -forms to finite open covers. To do so we rely on the previous results on Dirichlet subdomains and show that a certain space $\mathcal{S}$ of tensor products of bounded energy finite functions and indicators of open sets with zero-dimensional boundaries is dense in the space $\mathcal{H}$. The space $\mathcal{S}$ is considerably easier to handle than $\mathcal{H}$ itself, and using its denseness, we verify that the spaces of locally exact and locally harmonic forms are dense in $\mathcal{H}$ and $\mathcal{H}^{1}(X)$, respectively. In Section 5 these denseness results are employed to give the mentioned topological 
characterization for the nontriviality of the space $\mathcal{H}^{1}(X)$ in terms of the nontriviality of the first Čech cohomology $\check{H}^{1}(X)$. The potential theoretic condition needed here is that, roughly speaking, every set disconnecting a connected open set into two disjoint open pieces has positive capacity. Sufficient conditions for the validity of this capacity condition can be given in terms of irreducibility. For precise statements see Section 5. In Section [6 we first recall some notions of vector analysis proposed in 33 . Then we define the Hodge Laplacian (5) on 1-forms and prove that it yields a self-adjoint operator. We introduce harmonicity and observe that the space of harmonic 1 -forms equals $\mathcal{H}^{1}(X)$. Section 7 is devoted to the Navier-Stokes model. Here the main part of the necessary work lies in providing the preliminaries needed to make the model rigorous. In order to achieve this, we use local harmonicity and weighted energy measures to find a suitable substitute for the convection term. The mentioned statements on stationarity and nontriviality of solutions then follow naturally. The case of time-independent boundary conditions is elaborated on in Section 8 within the context of resistance forms.

To simplify notation, sequences or families indexed by the naturals will be written with the index set suppressed, e.g. $\left(a_{n}\right)_{n}$ stands for $\left(a_{n}\right)_{n \in \mathbb{N}}$. Similarly, $\lim _{n} a_{n}$ abbreviates $\lim _{n \rightarrow \infty} a_{n}$.

\section{SETUP AND PRELIMINARIES}

In this section we describe our setup in detail and briefly discuss some preliminary facts used in the sequel.

In our paper $(X, d)$ is assumed to be a connected topologically one-dimensional compact (hence separable) metric space. Furthermore, $m$ is assumed to be a finite Radon measure on $X$ such that $m(U)>0$ for any open set $U \subset X$. We finally assume that $(\mathcal{E}, \mathcal{F})$ is a symmetric strongly local regular Dirichlet form on $L_{2}(X, m)$ which has a spectral gap, i.e. there exists some $c>0$ such that for all $f \in \mathcal{F}$ we have

where

$$
\int_{X}\left(f-f_{X}\right)^{2} d m \leq c \mathcal{E}(f)
$$

$$
f_{X}=\frac{1}{m(X)} \int_{X} f d m
$$

With $\mathcal{E}_{1}(f, g):=\mathcal{E}(f, g)+\langle f, g\rangle_{L_{2}(X, m)}$ the space $\mathcal{F}$ becomes a Hilbert space. The notation

$$
\mathcal{B}:=\mathcal{F} \cap L_{\infty}(X, m)
$$

will be used to denote the space of $m$-a.e. bounded energy finite functions on $X$. Endowed with the norm $\|f\|_{\mathcal{B}}:=\mathcal{E}_{1}(f)^{1 / 2}+\operatorname{ess} \sup _{X}|f|$ it becomes a Banach algebra. In particular,

$$
\mathcal{E}(f, g) \leq\|f\|_{\mathcal{B}}\|g\|_{\mathcal{B}}, \quad f, g \in \mathcal{B} .
$$

Obviously $\mathcal{B}$ is dense in $\mathcal{F}$.

The regularity of $(\mathcal{E}, \mathcal{F})$ implies that for any $f, g \in \mathcal{B}$ there is a unique finite signed Radon measure $\Gamma(f, g)$ on $X$ such that

$$
2 \int_{X} \varphi d \Gamma(f, g)=\mathcal{E}(\varphi f, g)+\mathcal{E}(\varphi g, f)-\mathcal{E}(f g, \varphi)
$$

for all $\varphi \in C(X) \cap \mathcal{F}$. $\Gamma(f, g)$ is called the mutual energy measure of $f$ and $g$; cf. [15, 23, 44. The mapping $(f, g) \mapsto \Gamma(f, g)$ is symmetric and bilinear on $\mathcal{B}$. 
Furthermore, $\Gamma(f) \geq 0$ for any $f \in \mathcal{B}$. To define the energy measure of a general element $f \in \mathcal{F}$, let $\left(f_{n}\right)_{n} \subset \mathcal{B}$ be a sequence approximating $f$ in $\mathcal{F}$ and set

$$
\Gamma(f)(\varphi):=\lim _{n} \int_{X} \varphi d \Gamma\left(f_{n}\right), \quad \varphi \in C(X) \cap \mathcal{F} .
$$

Since

$$
\left|\left(\int_{X} \varphi d \Gamma\left(g_{1}\right)\right)^{1 / 2}-\left(\int_{X} \varphi d \Gamma\left(g_{2}\right)\right)^{1 / 2}\right| \leq\|\varphi\|_{L_{\infty}(X, m)}^{1 / 2} \mathcal{E}\left(g_{1}-g_{2}\right)^{1 / 2}
$$

for any $g_{1}, g_{2} \in \mathcal{B}$ and $\varphi \in C(X) \cap \mathcal{F}$ (cf. [23, Section 3.2]), the functional $\Gamma(f)$ is well defined. By regularity $\Gamma(f)$ extends to a positive linear functional on $C(X)$ and can be represented as

$$
\Gamma(f)(\varphi)=\int_{X} \varphi d \Gamma(f), \quad \varphi \in C(X),
$$

with a uniquely determined finite and nonnegative Radon measure $\Gamma(f)$ on $X$. By polarization we obtain mutual energy measures $\Gamma(f, g)$ for $f, g \in \mathcal{F}$, and clearly $\Gamma(f, g)(X)=\mathcal{E}(f, g)$. The Cauchy-Schwarz inequality

$$
|\Gamma(f, g)(A)| \leq \Gamma(f)(A)^{1 / 2} \Gamma(g)(A)^{1 / 2}
$$

for $f, g \in \mathcal{F}$ and $A \subset X$ Borel follows from standard arguments.

Remark 2.1. Another consequence of regularity together with our topological assumptions is that $C(X) \cap \mathcal{F}$ provides a special standard core for $\mathcal{E}$; i.e. for any compact set $K$ and any open set $U$ with $K \subset U$ there is a function $\varphi \in C(X) \cap \mathcal{F}$ such that $0 \leq \varphi \leq 1, \varphi \equiv 1$ on $K$ and $\varphi \equiv 0$ on $U^{c}$. See [23, Problem 1.4.1].

Let Cap denote the capacity corresponding to $(\mathcal{E}, \mathcal{F})$, given by

$$
\operatorname{Cap}(A)=\inf \left\{\mathcal{E}_{1}(u): u \in \mathcal{F}: u \geq 1 m \text {-a.e on } A\right\}
$$

for open sets $A \subset X$ and, for general, $B \subset U$,

$$
\operatorname{Cap}(B)=\inf \{\operatorname{Cap}(A): A \subset X \text { open, } B \subset A\}
$$

for general sets $B \subset X$. Any set of zero capacity is a null set for $m$. A statement is said to hold q.e. (quasi everywhere) on a subset $A \subset X$ if there exists some set $N \subset A$ with $\operatorname{Cap}(N)=0$, and the statement is valid for all $x \in A \backslash N$. A Borel function $f$ is said to be quasi-continuous if for any $\varepsilon>0$ there exists an open set $G \subset X$ such that $\operatorname{Cap}(G)<\varepsilon$ and $f$ is continuous on $X \backslash G$. Any function $f \in \mathcal{F}$, more precisely, any $m$-equivalence class $f$ of Borel functions in $\mathcal{F}$, possesses a Borel version (a representant of its $m$-equivalence class) $\tilde{f}$ which is quasi-continuous.

Let $\left(P_{t}\right)_{t>0}$ and $\left(G_{\alpha}\right)_{\alpha>0}$ be the semigroup of strongly continuous symmetric Markovian operators and the strongly continuous symmetric resolvent uniquely associated to $(\mathcal{E}, \mathcal{F})$. The semigroup $\left(P_{t}\right)_{t>0}$ associated with $(\mathcal{E}, \mathcal{F})$ is said to satisfy the absolute continuity condition if $A \mapsto P_{t} \mathbf{1}_{A}(x)$ is absolutely continuous with respect to $m$ for all $t>0$ and all $x \in X$. In other words, the associated transition kernels are assumed to be absolutely continuous.

Remark 2.2. If $\left(P_{t}\right)_{t>0}$ is a Feller semigroup (that is, as $X$ is compact, if each $P_{t}$ maps $C(X)$ into $C(X))$ and

$$
\left\|P_{t}\right\|_{L_{\infty}(X, m)} \leq B(t)\|f\|_{L_{1}(X, m)}
$$


for all $t>0$ and $f \in L_{1}(X, m)$ with some function $B:(0, \infty) \rightarrow(0, \infty)$, then it obviously satisfies the absolute continuity condition; cf. [13, p. 262].

To exclude further potential theoretic difficulties we make the following additional assumption.

Assumption 2.1. The semigroup $\left(P_{t}\right)_{t>0}$ satisfies the absolute continuity condition.

Definition 2.1. For an arbitrary set $B \subset X$, define

$$
\mathcal{F}_{B^{c}}:=\{f \in \mathcal{F}: \widetilde{f}=0 \text { q.e. on } B\} .
$$

The space $\mathcal{F}_{B^{c}}$ is a closed subspace of $\mathcal{F}$. Denote by $\mathcal{H}^{B}$ its orthogonal complement in $\mathcal{F}$ with respect to $\mathcal{E}_{1}$ and by $\mathcal{P}_{\mathcal{H}^{B}}$ the orthogonal projection onto $\mathcal{H}^{B}$. An element $h \in \mathcal{F}$ is called harmonic in $B^{c}$ in the Dirichlet form sense if $\mathcal{E}(h, \varphi)=0$ for all $\varphi \in C_{0}\left(B^{c}\right) \cap \mathcal{F}$. Every $h \in \mathcal{H}^{B}$ is harmonic in $B^{c}$, and if $B$ is closed, every function harmonic in $B^{c}$ in the Dirichlet form sense is an element of $\mathcal{H}^{B}$; cf. 23. Corollary 2.3.1].

We recall some properties of $\mathcal{P}_{\mathcal{H}^{B}}$ from [23] and a maximum principle. Under Assumption 2.1 both follow easily.

Proposition 2.1. Let $B \subset X$ be a Borel set.

(i) If for some $u \in \mathcal{F}$ and some constant $c \geq 0$ we have $|\widetilde{u}| \leq c$ q.e., then $\left|\left(\widetilde{\mathcal{P}_{\mathcal{H}^{B}} u}\right)\right| \leq$ c q.e. Moreover, $\left(\widetilde{\mathcal{P}_{\mathcal{H}^{B}} u}\right)=\widetilde{u}$ q.e. on $B$.

(ii) If $h: X \rightarrow \mathbb{R}$ is a q.e. bounded Borel function with m-equivalence class harmonic in $B^{c}$ in the Dirichlet form sense, then for q.e. $x \in B^{c}$ we have

$$
\inf _{y \in B} h(y) \leq h(x) \leq \sup _{y \in B} h(y) .
$$

Proposition 2.1 follows from known potential theoretic results, and we sketch them briefly in a probabilistic way. However, we would like to emphasize that probabilistic methods are not substantially used in the present paper.

By $Y=\left(Y_{t}\right)_{t \geq 0}$ we denote the $m$-symmetric Hunt process on $X$ uniquely associated with $(\mathcal{E}, \mathcal{F})$; cf. [15, 23, 56]. As $(\mathcal{E}, \mathcal{F})$ is local, $Y$ is a diffusion. The first hitting time of a Borel set $B \subset X$ by $Y$ is defined as

$$
\sigma_{B}:=\inf \left\{t>0: Y_{t} \in B\right\} \text {. }
$$

A set $N \subset X$ is called polar if there exists a Borel set $N_{1} \supset N$ such that

$$
\mathbb{P}_{x}\left(\sigma_{N_{1}}<\infty\right)=0
$$

for all $x \in X$. By Assumption 2.1 together with [23, Theorems 4.1.2 and 4.2.1] a set $N \subset X$ is polar if and only if $\operatorname{Cap}(N)=0$. To see Proposition 2.1 (i) consider $H_{B} f(x):=\mathbb{E}^{x}\left[e^{-\sigma_{B}} f\left(Y_{\sigma_{B}}\right)\right]$, well defined for any Borel function $f$. For $u \in \mathcal{F}$ the function $H_{B} \widetilde{u}$ is a quasi-continuous version of $\mathcal{P}_{\mathcal{H}^{B}} u$ by [23, Theorem 4.3.1]. Next, note that for any such $u$ we have

$$
H_{B} \widetilde{u}=\widetilde{u} \text { q.e. on } B \text {. }
$$

For 1-excessive functions (9) follows from [23, Lemma 4.3.1], in particular, (4.3.4). Recall that a Borel function $f$ is said to be 1-excessive if $f(x) \geq e^{-t} P_{t} f(x)$ and $f(x)=\lim _{t \rightarrow 0} e^{-t} P_{t} f(x)$ for $m$-a.a. $x \in X$. To verify (9) for general $u \in \mathcal{F}$ we may 
proceed as in the proof of [23, Theorem 4.3.1]: For any bounded Borel function $f$ on $X$ and any $\beta>0$ consider

$$
x \mapsto R_{\beta} f(x):=\mathbb{E}_{x} \int_{0}^{\infty} e^{-\beta t} f\left(Y_{t}\right) d t .
$$

$R_{\beta} f$ provides a quasi-continuous version of $G_{\beta} f$. For bounded $u$ and $\beta>0, R_{\beta} \widetilde{u}$ is the difference of two bounded 1-excessive functions and therefore $H_{B}\left(\beta R_{\beta} \widetilde{u}\right)=$ $\beta R_{\beta} \tilde{u}$ q.e. on $B$. By [23, Lemma 4.2.2 (ii)] there exists a set $N_{0}$ of zero capacity such that for all $x \in N_{0}^{c}$ we have $\lim _{\beta \rightarrow \infty} \beta R_{\beta} \widetilde{u}(x)=\widetilde{u}(x)$ and $\lim _{\beta \rightarrow \infty} H_{B}\left(\beta R_{\beta} \widetilde{u}\right)(x)$ $=H_{B} \widetilde{u}(x)$. For nonnegative $u \in \mathcal{F}$ identity (9) follows by cutting off and using monotone convergence and for general $u \in \mathcal{F}$ by considering $u \vee 0$ and $-u \vee 0$, both el-

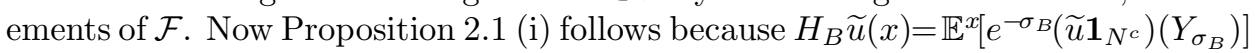
for any set $N$ that has zero capacity and therefore is polar. Concerning Proposition 2.1 (ii), note that [5, (the easier part of) Proposition 2.5] tells us that $h$ is harmonic in the probabilistic sense and therefore we have $h(x)=\mathbb{E}^{x}\left[h\left(Y_{t \wedge \tau_{D}}\right)\right]$ for any $t>0$, where $D$ is an arbitrary relatively open subset of $B^{c}$ and

$$
\tau_{D}:=\inf \left\{t \geq 0: Y_{t} \in D^{c}\right\},
$$

the first exit time of $Y$ from $D$. If $N$ is a set of zero capacity such that $|h|<c$ on $N^{c}$, then by the polarity of $N$ we have $h(x)=\mathbb{E}^{x}\left[\left(h \mathbf{1}_{N^{c}}\right)\left(Y_{t \wedge \tau_{D}}\right)\right]$, and the desired result follows using bounded convergence.

\section{Locally CONSTANT FUnCtions AND DiRichlet SUbDOMAins}

This section is concerned with Dirichlet subdomains $\mathcal{F}^{F} \subset \mathcal{F}$ that are constructed from functions that are locally constant on the neighborhood of a closed set $F$ of topological dimension zero. In Section 4 these subdomains will be used to obtain constructive descriptions for spaces of locally exact and locally harmonic 1 -forms on $X$. Since $X$ is topologically one-dimensional, every finite open cover has a finite refinement consisting of open sets with topologically zero-dimensional boundary. Recall that a compact subset of $X$ is topologically zero-dimensional if and only if it is a totally disconnected set; see e.g. [22]. The next lemma (and therefore also Proposition 3.1 below) uses this last fact in an essential way.

Lemma 3.1. Let $F \subset X$ be a compact set of topological dimension zero. For any $\delta>0$ there exists a finite collection $\mathcal{K}_{\delta}=\left\{K_{i}\right\}_{i=1}^{N}$ of disjoint compact sets $K_{i} \subset X$ of diameter less than $\delta$ such that $F=\bigcup_{i=1}^{N} K_{i}$.

Proof. There exists a finite $\frac{\delta}{2}$-net $\left\{x_{i}\right\}_{i=1}^{N}$ of points in $F$. Define $K_{1}$ as the set points of $F$ at the distance at most $\frac{\delta}{2}$ from $x_{1}$. Since $F$ is totally disconnected, $F \backslash K_{1}$ is compact. Hence we can define $K_{2}$ as the set points of $F \backslash K_{1}$ at the distance at most $\frac{\delta}{2}$ from $x_{2}$, and so on.

Let $F \subset X$ be closed. A function is said to be locally constant (q.e.) on an open cover of $F$ if it is constant (q.e.) on any connected component of the union of all sets in the cover. Consider the spaces

$$
\begin{aligned}
\mathcal{S}^{F}:=\left\{f \in \mathcal{F} \cap L_{\infty}(X, m)\right. & : \text { there exists a finite open cover of } F \\
& \text { such that } \tilde{f} \text { is locally constant q.e. on this cover }\}
\end{aligned}
$$


and

$$
\mathcal{S}_{c}^{F}:=C(X) \cap \mathcal{S}^{F} .
$$

The next proposition is technically the most involved result of this section.

Proposition 3.1. Let $F \subset X$ be a closed set of topological dimension zero. Then the space $\mathcal{S}^{F}$ is dense in $L_{2}(X, m)$.

Proof. We first consider $f \in C(X) \cap \mathcal{F}$. $X$ being compact, such a function $f$ is uniformly continuous on $X$. Let $\varepsilon>0$. Choose $\delta>0$ sufficiently small such that

$$
|f(p)-f(q)|<\varepsilon / 2 \quad \text { whenever } d(p, q)<\delta \text { for two points } p, q \in X .
$$

Let $\mathcal{K}_{\delta}=\left\{K_{i}\right\}_{i=1}^{N}$ be a finite partition of $F$ into compact sets $K_{i}$ of diameter less than $\delta$ according to Lemma 3.1. If $\varrho>0$ is the minimum distance between two of the sets $K_{i}$, let $0<\gamma<\delta \wedge \varrho / 3$,

$$
V_{i}:=\left\{x \in X: \operatorname{dist}\left(x, K_{i}\right)<\gamma / 2\right\}
$$

and

$$
W_{i}:=\left\{x \in X: \operatorname{dist}\left(x, K_{i}\right)<\gamma\right\},
$$

$i=1, \ldots, N$. Clearly the $W_{i}$ are disjoint and $\overline{V_{i}} \subset W_{i}$. We write $V:=\bigcup_{i=1}^{N} \overline{V_{i}}$ and $W:=\bigcup_{i=1}^{N} W_{i}$.

Choose nonnegative functions $\varphi_{i} \in C(X) \cap \mathcal{F}$ compactly supported in $W_{i}$, respectively, and such that

$$
\varphi_{i} \equiv \frac{1}{2}\left(\min _{p \in \overline{V_{i}}}|f(p)|+\max _{p \in \overline{V_{i}}}|f(p)|\right) \quad \text { on } \overline{V_{i}},
$$

$i=1, \ldots, N$. Let $\chi \in C(X) \cap \mathcal{F}$ be such that $0 \leq \chi \leq 1, \chi \equiv 1$ on $W^{c}$ and $\operatorname{supp} \chi \subset V^{c}$. Set $\varphi:=\chi f+\sum_{i=1}^{N} \varphi_{i} \in \mathcal{F}$ and $g:=\mathcal{P}_{\mathcal{H}^{V} \cup W^{c}} \varphi \in \mathcal{F}$, where we use notation from Definition [2.1. Then we have $\widetilde{g}=\varphi_{i}$ q.e. on each $\overline{V_{i}}$ by Proposition 2.1 (i) and therefore $g \in \mathcal{S}^{F}$. Similarly $\widetilde{g}=f$ q.e. on $W^{c}$. Again by Proposition 2.1 (i), the function $\widetilde{g}$ is q.e. bounded. Proposition 2.1 (ii) now implies

$$
\begin{aligned}
\min _{\overline{W_{i}}} f \leq \min \left\{\left.\varphi_{i}\right|_{\overline{V_{i}}}, \min _{\partial W_{i}} f\right\}= & \min _{q \in \partial W_{i} \cup \overline{V_{i}}} \widetilde{g}(q) \leq \widetilde{g}(p) \\
& \leq \max _{q \in \partial W_{i} \cup \overline{V_{i}}} \widetilde{g}(q)=\max \left\{\left.\varphi_{i}\right|_{\overline{V_{i}}}, \max _{\partial W_{i}} f\right\} \leq \max _{\overline{W_{i}}} f
\end{aligned}
$$

for q.e. $p \in W_{i} \backslash \overline{V_{i}}$ and any $i=1, \ldots, N$. By (10) and (12),

$$
\max _{\overline{W_{i}}} f-\min _{\overline{W_{i}}} f \leq \varepsilon
$$

for any $i$, and therefore $|f(p)-\widetilde{g}(p)| \leq \varepsilon$ for q.e. $p \in W \backslash V$, hence for q.e. $p \in X$. Consequently also $|f(p)-g(p)| \leq \varepsilon$ for $m$-a.a. $p \in X$. As $m$ is finite this implies the result because $C(X) \cap \mathcal{F}$ is uniformly dense in $C(X)$ and the latter is dense in $L_{2}(X, m)$.

Remark 3.1. It is not needed in our paper, but it also can be proved that if $\mathcal{F} \subset$ $C(X)$, then $\mathcal{S}_{c}^{F}$ is dense both in $L_{2}(X, m)$ and in $C(X)$ because $g \in \mathcal{S}_{c}^{F}$ such that the preceding estimates hold not just quasi-everywhere but everywhere. 
Now let $\mathcal{F}^{F}$ denote the $\mathcal{E}_{1}$-closure of $\mathcal{S}^{F}$. As $\mathcal{S}^{F}$ is dense in $L_{2}(X, m),\left(\mathcal{E}, \mathcal{F}^{F}\right)$ is a local Dirichlet form on $L_{2}(X, m)$. If $\mathcal{F} \subset C(X)$, then $\mathcal{S}_{c}^{F}$ is also dense in $L_{2}(X, m)$, and denoting its $\mathcal{E}_{1}$-closure by $\mathcal{F}_{c}^{F}$ we again obtain a local the Dirichlet form $\left(\mathcal{E}, \mathcal{F}_{c}^{F}\right)$, in this case even seen to be regular. In general the inclusion $\mathcal{F}^{F} \subset \mathcal{F}$ is proper. However, considering two disjoint closed zero-dimensional sets, the entire Dirichlet domain $\mathcal{F}$ can be reconstructed.

Theorem 3.1. Let $F_{1}$ and $F_{2}$ be closed topologically zero-dimensional subsets of $X$ and $F_{1} \cap F_{2}=\emptyset$. Then

$$
\mathcal{F}=\mathcal{F}^{F_{1}}+\mathcal{F}^{F_{2}}
$$

Remark 3.2. Here the subspaces $\mathcal{F}^{F_{1}}$ and $\mathcal{F}^{F_{2}}$ will typically have a nontrivial intersection, i.e. $\mathcal{F}$ will generally not be their direct sum. But of course we have

$$
\mathcal{F}^{F_{1}}+\mathcal{F}^{F_{2}}=\mathcal{F}^{F_{1}} \oplus\left(\mathcal{F}^{F_{2}} \cap\left(\mathcal{F}^{F_{1}}\right)^{\perp}\right),
$$

and the right hand side in this equality is an orthogonal, hence direct sum of two closed subspaces of $\mathcal{F}$.

The proof of the theorem relies on the following lemmas.

Lemma 3.2. Let $F_{1}$ and $F_{2}$ be disjoint closed subsets of $X$. Then there exists an open cover $\left\{U_{1}, U_{2}\right\}$ of $X$ such that $F_{1} \subset U_{1} \backslash \overline{U_{2}}$ and $F_{2} \subset U_{2} \backslash \overline{U_{1}}$.

Proof. Let $V_{1}$ and $V_{2}$ be disjoint open sets containing $F_{1}$ and $F_{2}$, respectively. $V_{1}^{c}$ and $F_{1}$ are disjoint, too, hence there exist disjoint open neighborhoods $W_{1}$ of $V_{1}^{c}$ and $W_{2}$ of $F_{1}$. Obviously $\left\{V_{1}, W_{1}\right\}$ covers $X$. Since $\overline{W_{1}} \cap W_{2}=\emptyset$, we have $F_{1} \subset V_{1} \backslash \overline{W_{1}}$. Since $\overline{V_{1}} \cap V_{2}=\emptyset, F_{2} \subset W_{1} \backslash \overline{V_{1}}$.

To any finite open cover we can associate an energy finite partition of unity:

Lemma 3.3. For any finite open cover $U_{1}, \ldots, U_{N}$ of $X$ there exist functions $\varphi_{i} \in$ $C(X) \cap \mathcal{F}, i=1, \ldots, N$, such that $0 \leq \varphi_{i} \leq 1, \operatorname{supp} \varphi_{i} \subset U_{i}$ and $\sum_{i=1}^{N} \varphi_{i}(x)=1$.

Proof. As $X$ is a normal space, we can find open sets $V_{1}, \ldots, V_{N}$ such that $\bar{V}_{i} \subset U_{i}$ for all $i$ and still $X=\bigcup_{i=1}^{N} V_{i}$; see e.g. [43, Proposition B.1]. For any $i$ let $\psi_{i} \in C(X) \cap \mathcal{F}$ be a function according to Remark 2.1 such that $\operatorname{supp} \psi_{i} \subset U_{i}$, $0 \leq \psi_{i} \leq 1$ and $\psi_{i} \equiv 1$ on $\overline{V_{i}}$. Now set $\varphi_{i}=\psi_{i}\left(\sum_{i=1}^{N} \psi_{i}\right)^{-1}$.

Given an open set $G \subset X$ we will regard $C_{0}(G)$ as a subset of $C(X)$ by trivial continuation. This simplifies notation in the next proofs.

Lemma 3.4. For any $F \subset X$ closed and zero-dimensional we have

$$
\mathcal{F}_{F^{c}} \subset \mathcal{F}^{F},
$$

where the left hand side is defined in Definition 2.1 .

Proof. By [23, Corollary 2.3.1], $\mathcal{F}_{F^{c}}$ equals the closure of $C_{0}\left(F^{c}\right) \cap \mathcal{F}$ in $\mathcal{E}_{1}$. For any $\varphi \in C_{0}\left(F^{c}\right) \cap \mathcal{F}$ we have $\operatorname{supp} \varphi \cap F=\emptyset$, hence there exist disjoint open neighborhoods of $\operatorname{supp} \varphi$ and $V$ of $F$. Since $\varphi \equiv 0$ on $V$, the function $\varphi$ is a member of $\mathcal{F}^{F}$.

We prove Theorem 3.1 
Proof. As $\mathcal{F}^{F_{1}}+\mathcal{F}^{F_{2}} \subset \mathcal{F}$ is obvious, it suffices to prove the converse inclusion. If $f \perp \mathcal{F}^{F_{1}}+\mathcal{F}^{F_{2}}$, then in particular $f \perp C_{0}\left(F_{1}^{c}\right) \cap \mathcal{F}$ and $f \perp C_{0}\left(F_{2}^{c}\right) \cap \mathcal{F}$ by the preceding lemma. But this implies $f \perp C(X) \cap \mathcal{F}$ : Let $\left\{U_{1}, U_{2}\right\}$ be an open cover of $X$ with $F_{1} \subset U_{1} \backslash \overline{U_{2}}$ and $F_{2} \subset U_{2} \backslash \overline{U_{1}}$ according to Lemma 3.2 and let $\varphi_{1}, \varphi_{2} \in C(X) \cap \mathcal{F}$ be such that $\operatorname{supp} \varphi_{i} \subset U_{i}$ and $\varphi_{1}(x)+\varphi_{2}(x)=1$ for all $x \in X$ as in Lemma 3.3. Given any $\varphi \in C(X) \cap \mathcal{F}$ we have $\varphi \varphi_{1} \in C_{0}\left(U_{1}\right) \cap \mathcal{F} \subset C_{0}\left(F_{2}^{c}\right) \cap \mathcal{F}$ and $\varphi \varphi_{2} \in C_{0}\left(U_{2}\right) \cap \mathcal{F} \subset C_{0}\left(F_{1}^{c}\right) \cap \mathcal{F}$. Therefore $f \perp \varphi_{1} \varphi$ and $f \perp \varphi_{2} \varphi$, which implies $f \perp \varphi=\varphi_{1} \varphi+\varphi_{2} \varphi$. Using regularity we finally obtain $f \perp \mathcal{F}$, which means $f=0$.

\section{LOCALLY EXACT AND LOCALLY HARMONIC 1-FORMS}

This section first recalls the definition of 1-forms and derivations based on Dirichlet forms as proposed by Cipriani and Sauvageot, [19,20, and then turns to related notions of local exactness and harmonicity.

In [19] and 20] the following construction of differential 1-forms has been developed. It may be considered for any symmetric local regular Dirichlet form on a locally compact second countable Hausdorff space. Endow the vector space $\mathcal{B} \otimes \mathcal{B}_{b}(X)$ of simple tensors with the symmetric bilinear form

$$
\langle a \otimes b, c \otimes d\rangle_{\mathcal{H}}=\int_{X} b d d \Gamma(a, c),
$$

$a \otimes b, c \otimes d \in \mathcal{B} \otimes \mathcal{B}_{b}(X)$. Let $\|\cdot\|_{\mathcal{H}}$ denote the associated norm and

$$
\text { ker }\|\cdot\|_{\mathcal{H}}:=\left\{\sum_{i} a_{i} \otimes b_{i} \in \mathcal{B} \otimes \mathcal{B}_{b}(X):\left\|\sum_{i} a_{i} \otimes b_{i}\right\|_{\mathcal{H}}=0\right\}
$$

(with finite sums). We write $\mathcal{H}$ for the completion of $\mathcal{B} \otimes \mathcal{B}_{b}(X) /$ ker $\|\cdot\|_{\mathcal{H}}$ with respect to $\|\cdot\|_{\mathcal{H}}$. Obviously $\mathcal{H}$ is a Hilbert space. We refer to it as the space of 1 -forms on $X$. The definitions

$$
c(a \otimes b):=(a c) \otimes b-c \otimes(a b)
$$

and

$$
(a \otimes b) d:=a \otimes(b d)
$$

for $a \otimes b \in \mathcal{B} \otimes \mathcal{B}_{b}(X), c \in \mathcal{B}$ and $d \in \mathcal{B}_{b}(X)$ extend continuously to uniformly bounded actions on $\mathcal{H}$ with

$$
\|c(a \otimes b)\|_{\mathcal{H}} \leq \sup _{X}|c|\|a \otimes b\|_{\mathcal{H}} \quad \text { and } \quad\|(a \otimes b) d\|_{\mathcal{H}} \leq \sup _{X}|d|\|a \otimes b\|_{\mathcal{H}},
$$

turning $\mathcal{H}$ into a bimodule. Using the locality of $(\mathcal{E}, \mathcal{F})$ it can be shown that the left and right action coincide; see for instance [30] or [40].

A derivation operator $\partial: \mathcal{B} \rightarrow \mathcal{H}$ can be defined by setting

$$
\partial f:=f \otimes \mathbf{1}
$$

It satisfies the Leibniz rule,

$$
\partial(f g)=f \partial g+g \partial f, \quad f, g \in \mathcal{B},
$$

and is a bounded linear operator satisfying

$$
\|\partial f\|_{\mathcal{H}}^{2}=\mathcal{E}(f), \quad f \in \mathcal{B} .
$$


On Euclidean domains and on smooth manifolds the operator $\partial$ coincides with the classical exterior derivative (in the $L_{2}$-sense). For more detailed information we refer the reader to [19, 20] and to the papers [18, 30, 31, 31, 33, 40], where this approach has been taken further in various respects.

Similarly as in [33, Section 2] we can extend the measure-valued bilinear mapping $\Gamma$ on $\mathcal{B}$ defined in (6) to a functional-valued bilinear mapping $\Gamma_{\mathcal{H}}$ on $\mathcal{H}$. Setting

$$
\Gamma_{\mathcal{H}}(a \otimes b, c \otimes d):=b d \Gamma(a, c)
$$

for simple tensors $a \otimes b, c \otimes d \in \mathcal{B} \otimes \mathcal{B}_{b}(X)$, we obtain a bilinear measure-valued map $\Gamma_{\mathcal{H}}$. Given a general element $u \in \mathcal{H}$ we may approximate it in $\mathcal{H}$ by a sequence $\left(u_{k}\right)_{k} \subset \mathcal{B} \otimes \mathcal{B}_{b}(X)$ of finite linear combinations of simple tensors and set

$$
\Gamma_{\mathcal{H}}(u)(\varphi):=\lim _{k} \int_{X} \varphi d \Gamma_{\mathcal{H}}\left(u_{k}\right)
$$

for any $\varphi \in \mathcal{B}_{b}(X)$. From (13) we easily obtain

$$
\left|\Gamma_{\mathcal{H}}(u)(\varphi)\right| \leq \sup _{x}|\varphi(x)|\|u\|_{\mathcal{H}}^{2},
$$

which defines a positive and bounded linear functional $\Gamma_{\mathcal{H}}(u)$ on $\mathcal{B}_{b}(X)$. For fixed $u \in \mathcal{H}$ we may extend $\Gamma_{\mathcal{H}}(u)$ to a generally unbounded bilinear functional on $L_{2}(X, m)$ by a simple approximation. By polarization $\Gamma_{\mathcal{H}}$ itself defines a bilinear mapping on $\mathcal{H}$.

Remark 4.1. Let $u \in \mathcal{H}$ be fixed. As $C(X) \subset \mathcal{B}_{b}(X)$, the Riesz representation theorem ensures the existence of a unique nonnegative Radon measure $\Gamma_{\mathcal{H}}(\omega)$ on $X$ such that for any

$$
\Gamma_{\mathcal{H}}(u)(\varphi):=\int_{X} \varphi d \Gamma_{\mathcal{H}}(u), \quad \varphi \in C(X) .
$$

Given a 1-form $\omega \in \mathcal{H}$, we refer to the support of the measure $\Gamma_{\mathcal{H}}(\omega)$ as the support of $\omega$; cf. [33, Section 2].

Lemma 4.1. If $f \in \mathcal{B}$ and $U \subset X$ open are such that $\operatorname{supp} f \subset U$, then also $\operatorname{supp} \partial f \subset U$.

Proof. By definition $\Gamma_{\mathcal{H}}(\partial f)=\Gamma(f)$. For arbitrary $V \subset X$ open, $\mathbf{1}_{V}$ may be approximated pointwise $m$-a.e. by a sequence $\left(\varphi_{n}\right)_{n} \subset C(X) \cap \mathcal{F}$ of functions $\varphi_{n}$ which are zero on $V^{c}$. If $V \subset U^{c} \subset(\operatorname{supp} f)^{c}$, then also $\operatorname{supp} \varphi_{n} \subset \bar{V} \subset U^{c}$, and therefore

$$
\Gamma(f)(V) \leq \liminf _{n} \int_{X} \varphi_{n} d \Gamma(f)=\liminf _{n}\left(2 \mathcal{E}\left(\varphi_{n} f, \varphi_{n}\right)-\mathcal{E}\left(f^{2}, \varphi_{n}\right)\right)=0
$$

by Fatou's lemma and the locality of $(\mathcal{E}, \mathcal{F})$.

Lemma 4.1 will be used in this section. Another application of $\Gamma_{\mathcal{H}}$ will be seen in Section 7

Recall that in the present paper we have assumed $X$ to be a compact, connected and topologically one-dimensional space. These assumptions have been utilized to prove the results of the previous section, which in turn now may be used to establish 
alternative descriptions of the space $\mathcal{H}$. Set

$$
\begin{aligned}
& \widetilde{\mathcal{H}}:=\operatorname{clos} \operatorname{span}\left\{g_{1} \otimes \mathbf{1}_{V_{1}}+g_{2} \otimes \mathbf{1}_{V_{2}}:\right. \\
& \qquad V_{i} \subset X \text { open with } \partial V_{i} \text { zero-dimensional, } \partial V_{1} \cap \partial V_{2}=\emptyset \\
& \left.\qquad g_{1} \in \mathcal{F}^{\partial V_{1}} \text { and } g_{2} \in \mathcal{F}^{\partial V_{2}} \cap\left(\mathcal{F}^{\partial V_{1}}\right)^{\perp}\right\} .
\end{aligned}
$$

Obviously $\widetilde{\mathcal{H}}$ is also a closed subspace of $\mathcal{H}$.

Theorem 4.1. The Hilbert spaces spaces $\widetilde{\mathcal{H}}$ and $\mathcal{H}$ coincide, $\widetilde{\mathcal{H}}=\mathcal{H}$.

The theorem is a consequence of the following two lemmas.

Lemma 4.2. Let $U \subset X$ be open and $f \in \mathcal{B}$. Then $f \otimes \mathbf{1}_{U} \in \tilde{\mathcal{H}}$.

Proof. We may assume $U \neq \emptyset$. Let $\left\{W_{n}\right\}_{n=1}^{\infty}$ be an exhaustion of $U$ by relatively compact open sets $W_{n}$, i.e. $\overline{W_{n}} \subset W_{n+1}$ and $U=\bigcup_{i=1}^{\infty} W_{n}$.

Let $n \in \mathbb{N}$ be fixed. For any $x \in \overline{W_{n}}$ let $V_{x}^{\prime}$ be a neighborhood of $x$ contained in $W_{n+1}$. As $X$ is topologically one-dimensional, there exists an open neighborhood $V_{x}$ of $x$ such that $\overline{V_{x}} \subset V_{x}^{\prime}$ and $\partial V_{x}$ is zero-dimensional. $\left\{V_{x}\right\}_{x \in \overline{W_{n}}}$ covers $\overline{W_{n}}$ and by compactness we can find a finite subcover $V_{x_{1}}, \ldots, V_{x_{N_{n}}}$ of $\overline{W_{n}}$. Set

$$
V^{n}:=\bigcup_{j=1}^{N_{n}} V_{x_{j}} .
$$

Clearly $\overline{W_{n}} \subset V^{n}$, and as the union is finite, we have $\overline{V^{n}} \subset \bigcup_{j=1}^{N_{n}} \overline{V_{x_{j}}}$ and $\partial V^{n} \subset$ $\bigcup_{j=1}^{N_{n}} \partial V_{x_{j}}$ for any fixed $n$. Consequently, $\partial V^{n}$ is zero-dimensional and

$$
\overline{V^{n}} \subset W_{n+1} \subset V^{n+1} .
$$

Accordingly $\bigcup_{n=1}^{\infty} V^{n}=U$ and by monotonicity also $\bigcup_{k=1}^{\infty} V^{2 k}=U$. For any $k$ the closed zero-dimensional sets $\partial V^{2 k}$ and $\partial V^{2 k+1}$ are disjoint. Theorem 3.1 yields the orthogonal decomposition

$$
\mathcal{F}=\mathcal{F}^{\partial V^{2 k}} \oplus \mathcal{F}^{\partial V^{2 k+1}} \cap\left(\mathcal{F}^{\partial V^{2 k}}\right)^{\perp},
$$

and therefore $f$ can be decomposed as

$$
f=f_{2 k}+f_{2 k+1}
$$

with $f_{2 k} \in \mathcal{F}^{\partial V^{2 k}}$ and $f_{2 k+1} \in \mathcal{F}^{\partial V^{2 k+1}} \cap\left(\mathcal{F}^{\partial V^{2 k}}\right)^{\perp}$. The 1-forms

$$
\omega_{k}:=f_{2 k} \otimes \mathbf{1}_{V^{2 k}}+f_{2 k+1} \otimes \mathbf{1}_{V^{2 k+1}}
$$

define a sequence $\left(\omega_{k}\right)_{k} \subset \widetilde{\mathcal{H}}$ which approximates $f \otimes \mathbf{1}_{U}$ weakly in $\mathcal{H}$ : For any finite linear combination

$$
\eta=\sum_{i=1}^{n} a_{i} \otimes b_{i}
$$


of simple tensors $a_{i} \otimes b_{i} \in \mathcal{B} \otimes \mathcal{B}_{b}(X)$, we have

$$
\begin{aligned}
\left\langle f \otimes \mathbf{1}_{U}-\right. & \left.\left(f_{2 k} \otimes \mathbf{1}_{V^{2 k}}+f_{2 k+1} \otimes \mathbf{1}_{V^{2 k+1}}\right), \eta\right\rangle_{\mathcal{H}} \\
= & \sum_{i=1}^{n}\left\langle f_{2 k} \otimes \mathbf{1}_{U \backslash V^{2 k}}+f_{2 k+1} \otimes \mathbf{1}_{U \backslash V^{2 k+1}}, a_{i} \otimes b_{i}\right\rangle_{\mathcal{H}} \\
= & \sum_{i=1}^{n}\left\langle f \otimes \mathbf{1}_{U \backslash V^{2 k}}-f_{2 k+1} \otimes \mathbf{1}_{V^{2 k+1} \backslash V^{2 k}}, a_{i} \otimes b_{i}\right\rangle_{\mathcal{H}} \\
= & \sum_{i=1}^{n} \int_{U \backslash V^{2 k}} b_{i} d \Gamma\left(f, a_{i}\right)-\sum_{i=1}^{n} \int_{V^{2 k+1} \backslash V^{2 k}} b_{i} d \Gamma\left(f_{2 k+1}, a_{i}\right) .
\end{aligned}
$$

As all $b_{i}$ are bounded and by the continuity properties of the finite measures $\Gamma\left(f, a_{i}\right)$, each summand in the first sum vanishes as $k$ goes to infinity. By Cauchy-Schwarz each summand of the second sum is bounded in modulus by

$$
\sup _{X}\left|b_{i}\right|\left|\Gamma\left(f_{2 k+1}, a_{i}\right)\left(V^{2 k+1} \backslash V^{2 k}\right)\right| \leq \sup _{X}\left|b_{i}\right| \Gamma\left(f_{2 k+1}\right)(X)^{1 / 2} \Gamma\left(a_{i}\right)\left(V^{2 k+1} \backslash V^{2 k}\right)^{1 / 2},
$$

which again converges to zero by the continuity of the $\Gamma\left(a_{i}\right)$, because

$$
\Gamma\left(f_{2 k+1}\right)(X)=\mathcal{E}\left(f_{2 k+1}\right) \leq \mathcal{E}(f)
$$

by the orthogonality in (21). As finite linear combinations of simple tensors are dense in $\mathcal{H}$ by construction, the weak convergence in $\mathcal{H}$ follows. By the Banach-Saks theorem there is a sequence $\left(k_{j}\right)$ such that the Cesaro averages

$$
\frac{1}{N} \sum_{j=1}^{N} \omega_{k_{j}},
$$

which are all elements of $\widetilde{\mathcal{H}}$, strongly converge to $f \otimes \mathbf{1}_{U}$ in $\mathcal{H}$ as $N$ goes to infinity.

Lemma 4.3. We have

$$
\mathcal{H}=\operatorname{clos} \operatorname{span}\left\{f \otimes \mathbf{1}_{U}: U \subset X \text { open and } f \in \mathcal{B}\right\} .
$$

This is a version of [30, Theorem 4.1]. The proof carries over from there.

Theorem 4.1 now follows, because Lemma 4.2 and Lemma 4.3 together imply $\mathcal{H} \subset \widetilde{\mathcal{H}}$.

Remark 4.2. Obviously the space

$$
\begin{aligned}
& \mathcal{S}:=\operatorname{span}\left\{g_{1} \otimes \mathbf{1}_{V_{1}}+g_{2} \otimes \mathbf{1}_{V_{2}}:\right. \\
& V_{i} \subset X \text { open with } \partial V_{i} \text { zero-dimensional, } \partial V_{1} \cap \partial V_{2}=\emptyset \text {, } \\
& \left.g_{1} \in \mathcal{F}^{\partial V_{1}} \text { and } g_{2} \in \mathcal{F}^{\partial V_{2}}\right\}
\end{aligned}
$$

is dense in $\widetilde{\mathcal{H}}$ and by Theorem 4.1 also in $\mathcal{H}$.

As $\partial$ is closed and $\mathcal{E}$ admits a spectral gap, the image $\operatorname{Im} \partial$ of $\mathcal{F}$ under the derivation $\partial$ is easily seen to be a closed linear subspace of the Hilbert space $\mathcal{H}$. This yields the orthogonal decomposition

$$
\mathcal{H}=\operatorname{Im} \partial \oplus \mathcal{H}^{1}(X),
$$

where we write $\mathcal{H}^{1}(X)$ to denote the orthogonal complement $(\operatorname{Im} \partial)^{\perp}$ of $\operatorname{Im} \partial$. For certain classes of fractal spaces (22) has been investigated in [18,20] and [40, and for harmonic spaces in [30]. We refer to the elements of Im $\partial$ as exact 1-forms. Clearly 
Im $\partial$ is nontrivial. Whether $\mathcal{H}^{1}(X)$ is nontrivial or not depends on the (global) topology of $X$; see 40 and Section 5 below. On the other hand (22) reminds us of the classical Hodge decomposition for differential forms on smooth one-dimensional manifolds, which is formulated in terms of local first order operators; cf. 41, 69]. The next definition introduces the key notions of local exactness and local harmonicity. They provide some 'localized' ways of testing whether a given 1-form belongs to $\operatorname{Im} \partial$ or $\mathcal{H}^{1}(X)$.

Definition 4.1. A 1 -form $\omega \in \mathcal{H}$ is called locally exact if there exist a finite open cover $\mathcal{U}=\left\{U_{\alpha}\right\}_{\alpha \in J}$ of $X$ and functions $f_{\alpha} \in \mathcal{B}, \alpha \in J$, such that

$$
\omega \mathbf{1}_{U_{\alpha}}=\partial f_{\alpha} \mathbf{1}_{U_{\alpha}}, \alpha \in J .
$$

A 1-form $\omega \in \mathcal{H}$ is called locally harmonic if there exist a finite open cover $\mathcal{U}=$ $\left\{U_{\alpha}\right\}_{\alpha \in J}$ of $X$ and functions $h_{\alpha} \in \mathcal{B}, \alpha \in J$, such that each $h_{\alpha}$ is harmonic on $U_{\alpha}$ (in the Dirichlet form sense) and

$$
\omega \mathbf{1}_{U_{\alpha}}=\partial h_{\alpha} \mathbf{1}_{U_{\alpha}}, \alpha \in J .
$$

These defining properties carry over to finite sums.

Lemma 4.4. Finite linear combinations $\omega=\sum_{i=1}^{N} \omega_{i}$ of locally exact (locally harmonic) 1-forms $\omega_{i}$ on $X$ are again locally exact (locally harmonic).

The simple proof by refinement is left to the reader.

Now let

and

$$
\mathcal{H}_{\text {loc }}:=\operatorname{clos} \operatorname{span}\{\omega \in \mathcal{H}: \omega \text { locally exact }\}
$$

$$
\mathcal{H}_{\text {loc }}^{1}:=\operatorname{clos} \operatorname{span}\{\omega \in \mathcal{H}: \omega \text { locally harmonic }\}
$$

denote the spaces of limits of locally exact and locally harmonic 1-forms on $X$, respectively. Obviously $\mathcal{H}_{l o c}^{1} \subset \mathcal{H}_{l o c} \subset \mathcal{H}$.

Lemma 4.5. The space $\mathcal{H}_{\text {loc }}^{1}$ is contained in $\mathcal{H}^{1}(X)$.

Proof. Given $\omega \in \mathcal{H}_{\text {loc }}^{1}$, let $\mathcal{U}=\left\{U_{\alpha}\right\}_{\alpha \in J}$ be a finite open cover of $X$ and $h_{\alpha} \in \mathcal{B}$ functions harmonic in $U_{\alpha}$, respectively, such that $\omega \mathbf{1}_{U_{\alpha}}=h_{\alpha} \mathbf{1}_{U_{\alpha}}$ for each $\alpha$. Now consider an arbitrary $f \in \mathcal{B}$. Let $\left\{\varphi_{\alpha}\right\}_{\alpha \in J} \subset C(X) \cap \mathcal{F}$ be an energy finite partition of unity subordinate to $\mathcal{U}$, i.e. $\varphi_{\alpha} \in C_{0}\left(U_{\alpha}\right) \cap \mathcal{F}, 0 \leq \varphi_{\alpha} \leq 1$ and $\sum_{\alpha \in J} \varphi_{\alpha}=$ 1 ; cf. Lemma 3.3. Then $\varphi_{\alpha} f \in \mathcal{B}, \operatorname{supp}\left(\varphi_{\alpha} f\right) \subset U_{\alpha}$ and by Lemma 4.1 also $\operatorname{supp}\left(\partial\left(\varphi_{\alpha} f\right)\right) \subset U_{\alpha}$ for any $\alpha$. Consequently, we have

$$
\begin{aligned}
\left\langle\partial\left(\varphi_{\alpha} f\right), \omega\right\rangle_{\mathcal{H}}=\left\langle\partial\left(\varphi_{\alpha} f\right), \partial h_{\alpha} \mathbf{1}_{U_{\alpha}}\right\rangle_{\mathcal{H}} & =\left\langle\partial\left(\varphi_{\alpha} f\right), \partial h_{\alpha}\right\rangle_{\mathcal{H}} \\
& =\mathcal{E}\left(\varphi_{\alpha} f, h_{\alpha}\right) \\
& =0,
\end{aligned}
$$

and summing over $\alpha \in J,\langle\partial f, \omega\rangle_{\mathcal{H}}=0$. As $f \in \mathcal{B}$ was arbitrary, $\omega$ must be an element of $(\operatorname{Im} \partial)^{\perp}=\mathcal{H}^{1}(X)$.

In fact, these spaces coincide.

\section{Theorem 4.2.}

(i) We have $\mathcal{H}_{\text {loc }}=\mathcal{H}$, i.e. the locally exact 1 -forms are dense in $\mathcal{H}$.

(ii) We have $\mathcal{H}_{\text {loc }}^{1}=\mathcal{H}^{1}(X)$, i.e. the locally harmonic 1 -forms are dense in $\mathcal{H}^{1}(X)$. 
To prove Theorem 4.2 we consider the spaces

$$
\mathcal{S}_{l o c}:=\operatorname{span}\left\{\partial f \mathbf{1}_{V}: V \subset X \text { open, } \partial V \text { zero-dimensional and } f \in \mathcal{S}^{\partial V}\right\}
$$
and

$$
\mathcal{S}_{\text {loc }}^{1}:=\mathcal{P}_{\mathcal{H}^{1}}\left(\mathcal{S}_{\text {loc }}\right),
$$

where $\mathcal{P}_{\mathcal{H}^{1}}$ denotes the orthogonal projection of $\mathcal{H}$ onto $\mathcal{H}^{1}(X)$. Similarly $\mathcal{P}_{\text {Im } \partial}$ denotes the orthogonal projection onto $\operatorname{Im} \partial$.

The next lemma is a key technical result of this section.

Lemma 4.6. Let $V \subset X$ be open with $\partial V$ zero-dimensional and $f \in \mathcal{S}^{\partial V}$. Then

(i) The 1-form $\partial f \mathbf{1}_{V}$ is locally exact.

(ii) The 1-form $\mathcal{P}_{\mathcal{H}^{1}}\left(\partial f \mathbf{1}_{V}\right)$ is locally harmonic.

Consequently $\mathcal{S}_{\text {loc }} \subset \mathcal{H}_{\text {loc }}$ and $\mathcal{S}_{\text {loc }}^{1} \subset \mathcal{H}_{\text {loc }}^{1}$.

Proof. Let $\left\{W_{k}\right\}_{k=1}^{N}$ be a finite open cover of $\partial V$ such that $f$ is constant on each $W_{k}$. Put

$$
W:=\bigcup_{k=1}^{N} W_{k}
$$

and define $U_{1}:=V \cup W$ as well as $U_{2}:=\bar{V}^{c} \cup W$. Obviously $\mathcal{U}=\left\{U_{1}, U_{2}\right\}$ is a finite open cover of $X$. By (23) we have

$$
\Gamma(f)(W) \leq \sum_{k=1}^{N} \Gamma(f)\left(W_{k}\right)=0 .
$$

Therefore $\left\|\partial f \mathbf{1}_{U_{1}}-\partial f \mathbf{1}_{V}\right\|_{\mathcal{H}}^{2}=\Gamma(f)\left(U_{1} \backslash V\right) \leq \Gamma(f)(W)=0$ and

$$
\left(\partial f \mathbf{1}_{V}\right) \mathbf{1}_{U_{1}}=\partial f \mathbf{1}_{V}=\partial f \mathbf{1}_{U_{1}} .
$$

Similarly

$$
\left(\partial f \mathbf{1}_{V}\right) \mathbf{1}_{U_{2}}=\left(\partial f \mathbf{1}_{U_{2}}\right) \mathbf{1}_{V}=\left(\partial f \mathbf{1}_{\bar{V}^{c}}\right) \mathbf{1}_{V}=0,
$$

and (i) follows. We turn to (ii). Let $g \in \mathcal{B}$ be a function such that

$$
\partial g=\mathcal{P}_{\text {Im } \partial}\left(\partial f \mathbf{1}_{V}\right) \text {. }
$$

Clearly $\omega:=\mathcal{P}_{\mathcal{H}^{1}}\left(\partial f \mathbf{1}_{V}\right)$ is a member of $\mathcal{H}^{1}(X)$. By (22) it equals $\partial f \mathbf{1}_{V}-\partial g$. According to (24),

$$
\omega \mathbf{1}_{U_{1}}=\left(\partial f \mathbf{1}_{V}-\partial g\right) \mathbf{1}_{U_{1}}=\partial(f-g) \mathbf{1}_{U_{1}} .
$$

Given $\varphi \in C_{0}\left(U_{1}\right) \cap \mathcal{F}$, we observe

$$
\mathcal{E}(\varphi, f-g)=\langle\partial \varphi, \partial(f-g)\rangle_{\mathcal{H}}=\langle\partial \varphi, \omega\rangle_{\mathcal{H}}=0
$$

by (24) and (22). Hence $f-g$ is harmonic on $U_{1}$. Similarly,

$$
\omega \mathbf{1}_{U_{2}}=\left(\partial f \mathbf{1}_{V}-\partial g\right) \mathbf{1}_{U_{2}}=-\partial g \mathbf{1}_{U_{2}}
$$

by (25), and for $\varphi \in C_{0}\left(U_{2}\right) \cap \mathcal{F}$ we have

$$
\mathcal{E}(\varphi, g)=-\langle\partial \varphi, \omega\rangle_{\mathcal{H}}=0,
$$

so $g$ is harmonic on $U_{2}$. Consequently, $\omega$ is locally harmonic.

\section{Lemma 4.7.}

(i) The space $\mathcal{S}_{\text {loc }}$ is dense in $\mathcal{H}$.

(ii) The space $\mathcal{S}_{\text {loc }}^{1}$ is dense in $\mathcal{H}^{1}(X)$. 
Proof. To prove (i) we will use the fact that $\widetilde{\mathcal{H}}=\mathcal{H}$. Let $V_{1}, V_{2} \subset X$ be open with $\partial V_{1}, \partial V_{2}$ zero-dimensional and disjoint. Recall Remark 4.2 and consider $g_{1} \otimes \mathbf{1}_{V_{1}}+$ $g_{2} \otimes \mathbf{1}_{V_{2}} \in \mathcal{S}$, where $g_{1} \in \mathcal{F}^{\partial V_{1}}$ and $g_{2} \in \mathcal{F}^{\partial V_{2}}$. Choose sequences $\left(g_{1}^{(n)}\right)_{n} \subset \mathcal{S}^{\partial V_{1}}$ and $\left(g_{2}^{(n)}\right)_{n} \subset \mathcal{S}^{\partial V_{2}}$ which approximate $g_{1}$ and $g_{2}$ in $\mathcal{E}_{1}$-norm, respectively. Then

$$
\begin{aligned}
\| g_{1} \otimes \mathbf{1}_{V_{1}} & +g_{2} \otimes \mathbf{1}_{V_{2}}-\left(g_{1}^{(n)} \otimes \mathbf{1}_{V_{1}}+g_{2}^{(n)} \otimes \mathbf{1}_{V_{2}}\right) \|_{\mathcal{H}}^{2} \\
& =\left\|\left(g_{1}-g_{1}^{(n)}\right) \otimes \mathbf{1}_{V_{1}}+\left(g_{2}-g_{2}^{(n)}\right) \otimes \mathbf{1}_{V_{2}}\right\|_{\mathcal{H}}^{2} \\
& =\Gamma\left(g_{1}-g_{1}^{(n)}\right)\left(V_{1}\right)+\Gamma\left(g_{1}-g_{1}^{(n)}\right)\left(V_{1} \cap V_{2}\right)+\Gamma\left(g_{2}-g_{2}^{(n)}\right)\left(V_{1} \cap V_{2}\right) \\
& +\Gamma\left(g_{2}-g_{2}^{(n)}\right)\left(V_{2}\right) \\
& \leq 2\left(\mathcal{E}_{1}\left(g_{1}-g_{1}^{(n)}\right)+\mathcal{E}_{1}\left(g_{2}-g_{2}^{(n)}\right)\right),
\end{aligned}
$$

which tends to zero as $n$ goes to infinity. By Lemma 4.6 the functions

$$
g_{1}^{(n)} \otimes \mathbf{1}_{V_{1}}+g_{2}^{(n)} \otimes \mathbf{1}_{V_{2}}
$$

are elements of $\mathcal{S}_{\text {loc }}$. As $\mathcal{S}$ itself is dense in $\widetilde{\mathcal{H}}=\mathcal{H}$, this implies (i). To see (ii), let $\eta \in \mathcal{H}^{1} \subset \mathcal{H}$. According to (i) there is a sequence $\left(\eta^{(n)}\right)_{n} \subset \mathcal{S}_{\text {loc }}$ approximating $\eta$ in $\mathcal{H}$. By (22) also $\left(\mathcal{P}_{\mathcal{H}^{1}}\left(\eta^{(n)}\right)\right)_{n}$ approximates $\eta$ in $\mathcal{H}$, and $\left(\mathcal{P}_{\mathcal{H}^{1}}\left(\eta^{(n)}\right)\right)_{n} \subset \mathcal{S}_{\text {loc }}^{1}$.

Now Theorem 4.2 follows from Lemma 4.6 and Lemma 4.7 because we have $\mathcal{H} \subset \mathcal{H}_{l o c}$ and $\mathcal{H}^{1} \subset \mathcal{H}_{l o c}^{1}$.

\section{NONTRiviality OF the FIRSt ČECH COHOMOLOGY}

The main results of this section are Theorems 5.1 and 5.2 below, which state that, roughly speaking, the (real) first Cech cohomology of $X$ is nontrivial if and only if $\mathcal{H}^{1}(X)$ is nontrivial.

For convenience and to fix notation we briefly recall some basic facts about Čech cohomology, [11, 21, 69]. Let $\mathcal{U}=\left\{U_{\alpha}\right\}_{\alpha \in J}$ be a finite open cover of $X$. To $\mathcal{U}$ we assign its $\check{C}$ ech complex which by definition is the abstract simplicial complex consisting of all $q$-simplices spanned by $q+1$ distinct elements $\alpha_{0}, \ldots, \alpha_{q}$ of $J$ for which

$$
U_{\alpha_{0} \cdots \alpha_{q}}:=U_{\alpha_{0}} \cap \cdots \cap U_{\alpha_{q}}
$$

is nonempty. We fix an orientation and write an ordered $q+1$ tuple $\alpha_{0} \cdots \alpha_{q}$ to denote an oriented $q$-simplex $\check{C}^{q}(\mathcal{U})$ that denotes the vector spaces of oriented Čech $q$-cochains, i.e. functions that assign a real number to each oriented $q$-simplex. A change of orientation of a simplex changes the sign of the function value on this simplex according to the sign of the corresponding permutation of the tuple. For our purposes $q=1$ is most important, therefore we restrict our attention to some special cases of more general facts.

The difference operator $d: \check{C}^{0}(\mathcal{U}) \rightarrow \check{C}^{1}(\mathcal{U})$ is defined by

$$
d f\left(\alpha_{0} \alpha_{1}\right):=f\left(\alpha_{1}\right)-f\left(\alpha_{0}\right), \quad f \in C^{0}(\mathcal{U}) .
$$

Its image $\check{B}^{1}(\mathcal{U}):=\operatorname{Im} d$ is a subspace of $C^{1}(\mathcal{U})$, the space of 1-coboundaries. Similarly, $d: \check{C}^{1}(\mathcal{U}) \rightarrow \check{C}^{2}(\mathcal{U})$ is defined by

$$
d f\left(\alpha_{0} \alpha_{1} \alpha_{2}\right):=f\left(\alpha_{1} \alpha_{2}\right)-f\left(\alpha_{0} \alpha_{2}\right)+f\left(\alpha_{0} \alpha_{1}\right), \quad f \in C^{1}(\mathcal{U}) .
$$


Its kernel $\check{Z}^{1}(\mathcal{U}):=$ ker $d$ is another subspace of $\check{C}^{1}(\mathcal{U})$, the space of 1 -cocycles. The quotient

$$
\check{H}^{1}(\mathcal{U}):=\check{Z}^{1}(\mathcal{U}) / \check{B}^{1}(\mathcal{U})
$$

is called the space of harmonic 1-cochains or first $\check{C}$ ech cohomology of $\mathcal{U}$. Given a cocycle $c \in \check{Z}^{1}(\mathcal{U})$, we denote its cohomology class in $\check{H}^{1}(\mathcal{U})$ by $[c]$. An open cover $\mathcal{V}=\left\{V_{\beta}\right\}_{\beta \in I}$ is a refinement of $\mathcal{U}=\left\{U_{\alpha}\right\}_{\alpha \in J}$, written $\mathcal{V}<\mathcal{U}$, if for each $\beta \in I$ there is some $\alpha=: \pi(\beta) \in J$ such that $V_{\beta} \subset U_{\alpha}$. This determines a refining map $\pi: I \rightarrow J$, which yields linear maps $\pi: \check{C}^{q}(\mathcal{U}) \rightarrow \check{C}^{q}(\mathcal{V})$ by

$$
\pi(c)\left(\beta_{0} \cdots \beta_{q}\right):=f\left(\pi\left(\beta_{0}\right) \cdots \pi\left(\beta_{q}\right)\right) .
$$

Since $d \circ \pi=\pi \circ d$, the maps $\pi$ themselves induce well-defined linear maps, often called refining maps:

$$
\pi_{\mathcal{U}}^{\mathcal{V}}: \check{H}^{1}(\mathcal{U}) \rightarrow \check{H}^{1}(\mathcal{V}), \quad \pi_{\mathcal{U}}^{\mathcal{V}}([c]):=[\pi(c)]
$$

We will make use of the following known fact and include a short textbook proof for convenience.

Lemma 5.1. The maps $\pi_{\mathcal{U}}^{\mathcal{V}}: \check{H}^{1}(\mathcal{U}) \rightarrow \check{H}^{1}(\mathcal{V})$ are injective.

Proof. Assume $c \in \mathcal{Z}^{1}(\mathcal{U})$ is such that $\pi(c)=b\left(\beta^{\prime}\right)-b(\beta) \in \check{B}^{1}(\mathcal{V})$ with $b \in \check{C}^{0}(\mathcal{V})$. Being an element of $\mathcal{Z}^{1}(\mathcal{U})=k e r d, c$ satisfies

$$
c\left(\pi(\beta) \pi\left(\beta^{\prime}\right)\right)=c\left(\alpha \pi\left(\beta^{\prime}\right)\right)-c(\alpha \pi(\beta)) .
$$

Therefore, if we set $h(\alpha, \beta):=b(\beta)-c(\alpha \pi(\beta))$ and define

$$
h(\alpha):=h\left(\alpha, \beta^{\prime}\right) \text { whenever } U_{\alpha} \cap V_{\beta} \neq \emptyset,
$$

$h$ is a well-defined element of $\check{C}^{0}(\mathcal{U})$. Obviously $d h=c$, and hence $c \in \check{B}^{1}(\mathcal{U})$.

Further, it can be shown that if both $\pi$ and $\sigma$ are refining maps, then $\sigma_{\mathcal{U}}^{\mathcal{V}}=\pi_{\mathcal{U}}^{\mathcal{V}}$. See [21] or 69. Therefore the spaces $\check{H}^{1}(\mathcal{U})$ together with the maps $\pi_{\mathcal{U}}^{\mathcal{U}}$ form a direct system along the set of open covers with the refinement relation $<$. The first $\check{C}$ ech cohomology $\check{H}^{1}(X)$ of the space $X$ is the corresponding direct limit

$$
\check{H}^{1}(X):=\underset{\mathcal{U}}{\lim } \check{H}^{1}(\mathcal{U}) .
$$

Recall that the direct limit on the right hand side can be obtained by considering the disjoint union $\bigsqcup_{\mathcal{U}} \check{H}^{1}(\mathcal{U})$ taken over all possible finite open covers $\mathcal{U}$ of $X$. Two of its elements $h_{1} \in \check{H}^{1}(\mathcal{U})$ and $h_{2} \in \check{H}^{1}(\mathcal{V})$ are equivalent, $h_{1} \sim h_{2}$, if there is a finite open cover $\mathcal{W}$ such that $\mathcal{W}<\mathcal{V}, \mathcal{W}<\mathcal{U}$ and $\pi_{\mathcal{U}}^{\mathcal{W}} h_{1}=\pi_{\mathcal{V}}^{\mathcal{W}} h_{2} \in \check{H}^{1}(\mathcal{W})$. The direct limit $\lim _{\mathcal{U}} \check{H}^{1}(\mathcal{U})$ then is defined as the resulting factor space $\left(\bigsqcup_{\mathcal{U}} \check{H}^{1}(\mathcal{U})\right) / \sim$.

A set $\mathbb{V}$ of open covers of $X$ is called cofinal if every open cover $\mathcal{U}$ of $X$ has a refinement $\mathcal{V} \in \mathbb{V}$. We record another simple fact.

Corollary 5.1. Let $\mathbb{V}$ be a cofinal set of open covers of $X$. If $\check{H}^{1}(X)$ is nontrivial, then for some $\mathcal{V} \in \mathbb{V}$ the space $\check{H}^{1}(\mathcal{V})$ must be nontrivial.

Proof. Assume that $\check{H}^{1}(\mathcal{V})=\{0\}$ for all $\mathcal{V} \in \mathbb{V}$. For any $h \in \bigsqcup_{\mathcal{U}} \check{H}^{1}(\mathcal{U})$ there is some open cover $\mathcal{W}$ of $X$ such that $h \in \check{H}^{1}(\mathcal{W})$. As $\mathbb{V}$ is cofinal, $\mathcal{W}$ has a refinement $\mathcal{V} \in \mathbb{V}$. By the above assumption, $\pi_{\mathcal{W}}^{\mathcal{V}} h=0$ in $\check{H}^{1}(\mathcal{V})$. If $\mathcal{U}$ is any further refinement of $\mathcal{V}$, then

$$
\pi_{\mathcal{V}}^{\mathcal{U}} h=\pi_{\mathcal{V}}^{\mathcal{U}} \pi_{\mathcal{W}}^{\mathcal{V}} h=0
$$


which implies that the equivalence class of $h$ in $\check{H}^{1}(X)$ is zero. As $h$ was arbitrary, this argument would imply $\check{H}^{1}(X)=\{0\}$, contradicting the assumption of the lemma.

Now let $\mathbb{V}_{0}$ be the set of all finite open covers $\mathcal{V}=\left\{V_{\beta}\right\}_{\beta \in I}$ such that

all open sets $V_{\beta}$ are connected and such that

$$
\text { for distinct } \beta, \beta^{\prime}, \beta^{\prime \prime} \in I \text { we have } V_{\beta} \cap V_{\beta^{\prime}} \cap V_{\beta^{\prime \prime}}=\emptyset \text {. }
$$

The one-dimensionality of $X$ implies the following.

Lemma 5.2. The set $\mathbb{V}_{0}$ of such covers is cofinal.

Proof. As $X$ has Lebesgue covering dimension one, an arbitrary open cover of $X$ has a refinement satisfying the intersection property in (26). Considering the connected components of the sets contained in this refinement, we obtain a cover by connected open sets still satisfying the intersection property. Since $X$ is compact, finitely many of these sets suffice to cover $X$. Consequently, any open cover of $X$ has a refinement in $\mathbb{V}_{0}$.

To prove the theorems below we use the following characterization of exact 1forms.

Proposition 5.1. Let $U \subset X$ be open and $f \in \mathcal{B}$. Then $f \otimes \mathbf{1}_{U}$ is exact if and only if there exists a function $g \in \mathcal{B}$ such that $\widetilde{f}=\widetilde{g}$ q.e. on $U$ and $\widetilde{g}$ is constant q.e. on each connected component of $X \backslash U$.

Proof. The "if" part is clear. For the converse implication, assume there is some $g \in \mathcal{B}$ that equals $\left.f\right|_{U}$ on $U$ q.e. and is constant q.e. on each connected component of $X \backslash U$. Then

$$
\begin{aligned}
\left\|h \otimes \mathbf{1}_{U}-g \otimes \mathbf{1}\right\|_{\mathcal{H}}^{2} & =\left\|(h-g) \otimes \mathbf{1}_{U}+g \otimes \mathbf{1}_{X \backslash U}\right\|_{\mathcal{H}}^{2} \\
& =\Gamma(h-g)(U)+\Gamma(g)(X \backslash U) \\
& =0 .
\end{aligned}
$$

The first main result of this section is the following.

Theorem 5.1. Let $X$ be compact and topologically one-dimensional. If $\mathcal{H}^{1}(X)$ is nontrivial, then $\breve{H}^{1}(X)$ is nontrivial.

Proof. Assume $\check{H}^{1}(X)$ is trivial. Let $\partial f \mathbf{1}_{V} \in \mathcal{S}_{l o c}$ be arbitrary. Recall that according to the definition of $\mathcal{S}_{l o c}$, the set $V \subset X$ is open and has a zero-dimensional boundary $\partial V$ admitting a finite open cover on which $f$ is locally constant. $\partial V$ being zero-dimensional, the cover admits a finite refinement consisting of disjoint open sets $U_{1}, \ldots, U_{N}$.

Put $V^{\prime}:=V \cup \bigcup_{i=1}^{N} U_{i}$. We claim that $\left(V^{\prime}\right)^{c}$ and $\bar{V}$ are disjoint. In fact, we have $\partial V \subset \bigcup_{i=1}^{N} U_{i}$, and the sets $\partial V$ and $\partial\left(\bigcup_{i=1}^{N} U_{i}\right) \subset \bigcup_{i=1}^{N} \partial U_{i}$ are disjoint: If there were some $x \in \partial V \cap \partial U_{i}$, then there had to be some $k \neq i$ such that $x \in U_{k}$, resulting in $U_{i} \cap U_{k} \neq \emptyset$, which is impossible. Accordingly, $\partial V^{\prime}$ and $\partial V$ are disjoint, which implies our previous claim.

Let $W$ be an open neighborhood of $\left(V^{\prime}\right)^{c}$ such that $W \cap V=\emptyset$. Then $\mathcal{U}:=$ $\left\{V, W, U_{1}, \ldots, U_{N}\right\}$ is an open cover of $X$. As a consequence of Lemma 5.1, $\check{H}^{1}(\mathcal{U})$ must be trivial. Since by construction no more than two sets of $\mathcal{U}$ have nonempty 
intersection, this means its Čech complex is a graph and does not contain a cycle, i.e. it is a tree. Therefore the sets $U_{1}, \ldots, U_{N}$ belong to different connected components of $X \backslash V$. But then $\tilde{f}$ possesses a continuation to all of $X$ which is constant q.e. on each of these components, and according to Proposition 5.1 $\partial f \mathbf{1}_{V}=f \otimes \mathbf{1}_{V}$ is a gradient.

Since $\partial f \mathbf{1}_{V}$ was an arbitrary element of $\mathcal{S}_{l o c}$, any member of the latter space is a gradient, and its closure is $\operatorname{Im} \partial=\mathcal{H}$, leaving $\mathcal{H}^{1}=\{0\}$.

Our proof of the converse implication needs an additional assumption. Let $U \subset$ $X$ be a connected open set. A set $D \subset U$ will be called disconnecting for $U$ if $U$ decomposes into a disjoint union

$$
U=U_{1} \cup U_{2} \cup D
$$

with $U_{1}$ and $U_{2}$ open. In other words, the removal of $D$ turns the connected set $U$ into the disconnected set $U_{1} \cup U_{2}$. If $\mathbb{V}$ is a set of covers consisting of connected open sets, a set $D$ will be called disconnecting for $\mathbb{V}$ if it is disconnecting for any connected open set in any of the covers of $\mathbb{V}$.

Theorem 5.2. Let $X$ be compact and topologically one-dimensional. Assume that there exists a cofinal set $\mathbb{V}$ of finite open covers $\mathcal{V}=\left\{V_{\beta}\right\}_{\beta \in I}$ satisfying (26) and such that any set $D$ which is disconnecting for $\mathbb{V}$ has positive capacity, $\operatorname{Cap}(D)>0$. Then if $\check{H}^{1}(X)$ is nontrivial, $\mathcal{H}^{1}(X)$ must also be nontrivial.

\section{Remark 5.1.}

(i) If $(\mathcal{E}, \mathcal{F})$ is a resistance form in the sense of Kigami $48,50,51$, then points have positive capacity and so the hypothesis in Theorem 5.2 obviously holds. This is the case if, for instance, the spectral dimension $d_{S}$ exists and is less than 2 .

(ii) Theorem 5.2 also applies to certain classes of self-similar and cell-structured sets which carry a diffusion that admits transition densities. Examples include generalized Sierpinski carpets [4,5. Note that in these cases the spectral dimension $d_{S}$ may even be greater than or equal to 2 .

Proof. By Corollary 5.1 there is a cover $\mathcal{V} \in \mathbb{V}$ such that $\check{H}^{1}(\mathcal{V})$ is nontrivial. The Cech graph of this cover $\mathcal{V}$ must contain a cycle; i.e. there must be covering sets $V_{\beta_{0}}, V_{\beta_{1}}, \ldots, V_{\beta_{N}} \in \mathcal{V}$ such that $V_{\beta_{i}} \cap V_{\beta_{i+1}} \neq \emptyset, i=0, \ldots, N$, where $\beta_{N+1}:=\beta_{0}$. We may assume

$$
V_{\beta_{0}} \cap \overline{V_{\beta_{0} \beta_{1}}} \cap \overline{V_{\beta_{0} \beta_{N}}}=\emptyset .
$$

For if $x_{1} \in V_{\beta_{0}} \cap \overline{V_{\beta_{1}}} \cap \overline{V_{\beta_{N}}}$, then there is a base set $V_{1}$ containing $x$ and such that $\overline{V_{1}} \subset V_{\beta_{0}}$. If now $V_{\beta_{0}} \cap \overline{V_{\beta_{1}} \backslash V_{1}} \cap \overline{V_{\beta_{N}}}=\emptyset$, put $V_{2}:=\emptyset$. If there is some $x_{2} \in V_{\beta_{0}} \cap \overline{V_{\beta_{1}} \backslash V_{1}} \cap \overline{V_{\beta_{N}}}$, choose another base set $V_{2}$ containing $x_{2}$ and such that $\overline{V_{2}} \subset V_{\beta_{0}}$. Now proceed further by induction: If $V_{\beta_{0}} \cap \overline{V_{\beta_{1}} \backslash \bigcup_{j=1}^{k} V_{k}} \cap \overline{V_{\beta_{N}}}=\emptyset$, then put $V_{k+1}=\emptyset$, and if a point $x_{k+1}$ is contained, choose a base set $V_{k+1}$ containing $x_{k+1}$ and such that $\overline{V_{k+1}}$ is in $V_{\beta_{0}}$. Finally replace $V_{\beta_{1}}$ by $V_{\beta_{1}} \backslash \bigcup_{j=1}^{\infty} V_{j}$ and denote it again by $V_{\beta_{1}}$. As any point of the space must be contained in one of the base sets, (28) now holds. On the other hand the new system of open sets still covers $X$. Note also that this modification does not alter the set $\left(\bigcup_{k=1}^{N} V_{\beta_{k}}\right) \backslash V_{\beta_{0}}$.

We have

$$
\partial V_{\beta_{0}} \cap \overline{V_{\beta_{0} \beta_{1}}} \cap \overline{V_{\beta_{0} \beta_{N}}}=\emptyset .
$$


Clearly the set $\partial V_{\beta_{0}} \cap V_{\beta_{0} \beta_{1}} \cap \overline{V_{\beta_{0} \beta_{N}}}$ is empty. For any $x \in \partial V_{\beta_{0}} \cap \partial V_{\beta_{0} \beta_{1}} \cap V_{\beta_{0} \beta_{N}}$ we could find an open neighborhood $V_{x} \subset V_{\beta_{0} \beta_{2}}$ which intersects $V_{\beta_{0} \beta_{2}}$, which contradicts (26). Any $x \in \partial V_{\beta_{0}} \cap \partial V_{\beta_{0} \beta_{1}} \cap \partial V_{\beta_{0} \beta_{N}}$ would have to be contained in some other set $V_{\beta^{\prime}}$ of the cover, causing a similar contradiction.

Clipping (28) and (29), $\overline{V_{\beta_{0} \beta_{1}}}$ and $\overline{V_{\beta_{0} \beta_{N}}}$ are seen to be disjoint compact subsets of $X$. Therefore we can find an open set $W \supset \overline{V_{\beta_{0} \beta_{1}}}$, disjoint from $\overline{V_{\beta_{0} \beta_{N}}}$, and a function $\varphi \in C_{0}(X) \cap \mathcal{F}$ such that $0 \leq \varphi \leq 1, \varphi \equiv 1$ on $\overline{V_{\beta_{0} \beta_{1}}}$ and zero outside $W$. In particular, $\varphi \equiv 1$ on $\partial V_{\beta_{0}} \cap V_{\beta_{1}}$ and $\varphi \equiv 0$ on $\partial V_{\beta_{0}} \cap V_{\beta_{N}}$. These two sets belong to the same connected component $K$ of $X \backslash V_{\beta_{0}}$.

Now assume there is a function $f \in \mathcal{B}$ such that for its quasi-continuous Borel version $\widetilde{f}$ we have $\widetilde{f}=\varphi$ q.e. on $V_{\beta_{0}}$ and $\widetilde{f}$ is constant q.e. on any connected component of $X \backslash U$. Then there exist a set $N \subset \bigcup_{i=1}^{N} V_{\beta_{k}}$ of zero capacity and a constant $c \in \mathbb{R}$ such that $\tilde{f} \equiv c$ on $\bigcup_{i=1}^{N} V_{\beta_{k}} \backslash\left(V_{\beta_{0}} \cup N\right), \tilde{f} \equiv 1$ on $V_{\beta_{0} \beta_{1}} \backslash N$ and $\tilde{f} \equiv 0$ on $V_{\beta_{0} \beta_{N}}$. In particular, $\tilde{f}$ must be discontinuous on at least one of the sets $\left(\partial V_{\beta_{0}} \cap V_{\beta_{1}}\right) \backslash N$ and $\left(\partial V_{\beta_{0}} \cap V_{\beta_{N}}\right) \backslash N$. As the sets $\partial V_{\beta_{0}} \cap V_{\beta_{1}}$ and $\partial V_{\beta_{0}} \cap V_{\beta_{N}}$ are disconnecting (for $V_{\beta_{1}}$ and $V_{\beta_{N}}$, respectively), they both are of positive capacity, which contradicts the quasi-continuity of $\widetilde{f}$.

Consequently, $\partial \varphi \mathbf{1}_{V_{\beta_{0}}}$ is not a gradient but a nontrivial element of $\mathcal{H}^{1}$.

A coarse sufficient condition for the validity of a capacity condition in Theorem 5.2 can be formulated in terms of the irreducibility of restricted Dirichlet forms. Given a nonempty open subset $U \subset X$, let $\mathcal{F}^{U}$ denote the $\mathcal{E}_{1}$-closure of $C_{0}(U) \cap \mathcal{F}$ and write

$$
\mathcal{E}^{U}:=\left.\mathcal{E}\right|_{\mathcal{F}^{U}}
$$

Then $\left(\mathcal{E}^{U}, \mathcal{F}^{U}\right)$ is a regular Dirichlet form, referred to as the restriction of $(\mathcal{E}, \mathcal{F})$ to $U$ (with Dirichlet boundary conditions). The associated strongly continuous symmetric Markovian semigroup on $L_{2}(U, m)$ is denoted by $\left(P_{t}^{U}\right)_{t \geq 0}$, often called the killed semigroup on $U$. The symmetric Hunt process associated with $\left(\mathcal{E}^{U}, \mathcal{F}^{U}\right)$ and $\left(P_{t}^{U}\right)_{t \geq 0}$ is $\left(Y_{t}^{U}\right)_{t \geq 0}$, defined by

$$
Y_{t}^{U}:=\left\{\begin{array}{l}
Y_{t}, t<\tau_{U}, \\
\Delta, t \geq \tau_{U},
\end{array}\right.
$$

where $\Delta$ is the point at infinity in the one-point compactification of $X$ or, if $X$ is already compact, an adjoined isolated point. A function $f$ on $X$ or a subset of $X$ is set to be zero at $\Delta, f(\Delta):=0$; cf. [17,23]. A Borel set $A \subset U$ is an invariant set for $\left(\mathcal{E}^{U}, \mathcal{F}^{U}\right)$ if

$$
P_{t}^{U}\left(\mathbf{1}_{A} f\right)(x)=0 \text { for } m \text {-a.e. } x \in U \backslash A
$$

for any $f \in L_{2}(U, m)$ and $t>0$. $\left(\mathcal{E}^{U}, \mathcal{F}^{U}\right)$ is called irreducible if every invariant subset $A$ for $\left(\mathcal{E}^{U}, \mathcal{F}^{U}\right)$ is trivial, i.e. if $m(A)=0$ or $m(U \backslash A)=0$. For $(\mathcal{E}, \mathcal{F})$ itself invariance and irreducibility are defined in an analogous manner. See [15, 23] for further details.

For $U \subset X$ open let $\operatorname{Cap}^{U}$ denote the capacity with respect to $\left(\mathcal{E}^{U}, \mathcal{F}^{U}\right)$ in the sense of (7), that is,

$$
\operatorname{Cap}^{U}(A)=\inf \left\{\mathcal{E}_{1}(f): f \in \mathcal{F}^{U}: \tilde{f} \geq 1 m \text {-a.e on } A\right\}
$$


for $A \subset U$ open, and for general $B \subset U$,

$$
\operatorname{Cap}^{U}(B)=\inf \left\{\operatorname{Cap}^{U}(A): A \subset U \text { open, } B \subset A\right\} \text {. }
$$

For a Borel set $B \subset U$ let $\sigma_{B}^{U}$ denote the first hitting time of $B$ by the restricted process $Y^{U}$,

$$
\sigma_{B}^{U}=\inf \left\{t>0: Y_{t}^{U} \in B\right\} .
$$

Lemma 5.3. Let $B \subset U$ be a Borel set. Then $\operatorname{Cap}^{U}(B)>0$ implies $\operatorname{Cap}(B)>0$.

Proof. As $(\mathcal{E}, \mathcal{F})$ satisfies the absolute continuity hypothesis by Assumption 2.1, so does $\left(\mathcal{E}^{U}, \mathcal{F}^{U}\right)$. By [23, Theorems 4.1.2 and 4.2.1] $B$ cannot be polar for $Y^{U}$, that is, $\mathbb{P}_{x}\left(\sigma_{B}^{U}<\infty\right)>0$ for some $x \in U$. Then clearly also $\mathbb{P}_{x}\left(\sigma_{B}<\infty\right)>0$, which means that $B$ is not polar for $Y$, and the result follows by another application of the cited theorems.

We obtain the following consequences of irreducibility.

Proposition 5.2. If $U \subset X$ is a connected open set and $\left(\mathcal{E}^{U}, \mathcal{F}^{U}\right)$ is irreducible, then any set $D$ disconnecting $U$ has positive capacity.

Proof. Assume that $U=U_{1} \cup U_{2} \cup D$ with $U_{1}, U_{2}$ open and the union being disjoint. We may assume $m(D)=0$ because $m$ charges no set of zero capacity. Since $m\left(U_{1}\right)>0$ and $m\left(U_{2}\right)>0$, neither $U_{1}$ nor $U_{2}$ can be invariant for $\left(\mathcal{E}^{U}, \mathcal{F}^{U}\right)$. Hence without loss of generality there exist some $t>0, f \in L_{2}(U, m)$ with $f \geq 0$ and some set $A \subset U_{1}$ with $m(A)>0$ such that

$$
\mathbb{E}_{x}\left[\left(\mathbf{1}_{U_{2}} f\right)\left(Y_{t}^{U}\right)\right]=P_{t}^{U}\left(\mathbf{1}_{U_{2}} f\right)(x)>0 \text { for all } x \in A .
$$

In particular, for all $x \in A$ the $\mathbb{P}_{x}$-probability to have a connected path $Y^{U}([0, t])$ joining $x$ and $U_{2}$ is positive. Each such path necessarily hits the relative boundary of $U_{2}$ with respect to $U$, and this relative boundary is contained in $D$. If $\operatorname{Cap}^{U}(D)=0$, then $D$ must be polar for $Y^{U}$, and in particular,

$$
\mathbb{P}_{x}\left(Y_{t} \in U_{2}\right) \leq \mathbb{P}_{x}\left(\sigma_{D}^{U}<\infty\right)=0 \text { for all } x \in A,
$$

a contradiction. Consequently $\operatorname{Cap}^{U}(D)>0$, and by Lemma 5.3 the conclusion follows.

Corollary 5.2. Assume that there exists a cofinal set $\mathbb{V}$ of finite open covers $\mathcal{V}=$ $\left\{V_{\beta}\right\}_{\beta \in I}$ satisfying (26) and such that for any open set $V_{\beta}$ from any cover $\mathcal{V}$ of $\mathbb{V}$, the Dirichlet form $\left(\mathcal{E}^{V_{\beta}}, \mathcal{F}^{V_{\beta}}\right)$ is irreducible. Then the conclusion of Theorem 5.2 holds.

\section{Form LAPLACIAN AND HARMONIC 1-FORMS}

In this section we first recall some notions of vector calculus as proposed in 33 . Then we give definitions for the Hodge Laplacian on 1-forms and for harmonic 1forms. Finally, we define a specific functional needed in the next section to formulate Navier-Stokes type equations.

Due to the self-duality of $\mathcal{H}$ we regard the elements of $\mathcal{H}$ also as vector fields and $\partial$ as a generalization of the classical gradient operator. It may be viewed as an unbounded closed linear operator from $L_{2}(X, m)$ to $\mathcal{H}$ with domain $\mathcal{F}$. Let $\mathcal{B}^{*}$ denote the dual of $\mathcal{B}$ with the usual norm

$$
\|u\|_{\mathcal{B}^{*}}=\sup \left\{|u(f)|: f \in \mathcal{B},\|f\|_{\mathcal{B}} \leq 1\right\} .
$$


Given a vector field of form $g \partial f$, its divergence can be defined similarly as in 33 . by

$$
\partial^{*}(g \partial f):=-\int_{X} g d \Gamma(\cdot, f) \in \mathcal{B}^{*} .
$$

The map $g \partial f \mapsto \partial^{*}(g \partial f)$ extends continuously to a bounded linear operator $\partial^{*}$ from $\mathcal{H}$ into $\mathcal{B}^{*}$, as shown in [33, Lemma 3.1]. Note that this is a definition in a distributional sense. Seen as an unbounded operator from $\partial^{*}: \mathcal{H} \rightarrow L_{2}(X, m)$ with domain

$\operatorname{dom} \partial^{*}:=\left\{v \in \mathcal{H}:\right.$ there exists $v^{*} \in L_{2}(X, m)$ such that

$$
\left.\left\langle u, v^{*}\right\rangle_{L_{2}(X, m)}=-\langle\partial u, v\rangle_{\mathcal{H}} \text { for all } u \in \mathcal{F}\right\},
$$

the operator $-\partial^{*}$ is seen to be the adjoint of $\partial$, i.e.

$$
\left\langle u, \partial^{*} v\right\rangle_{L_{2}(X, m)}=-\langle\partial u, v\rangle_{\mathcal{H}}, \quad u \in \mathcal{F} .
$$

Sometimes $\partial$ is referred to as the codifferential associated with $\partial$. Its domain dom $\partial^{*}$ is dense in $\mathcal{H}$. Let $A$ denote the infinitesimal generator of $(\mathcal{E}, \mathcal{F})$. For any $f \in \mathcal{B}$ we have

$$
A f=\partial^{*} \partial f
$$

in $\mathcal{B}^{*}$ (see [33, Lemma 3.2]), and for functions $f$ from the domain $\operatorname{dom} A$ of $A$ the identity (32) holds in $L_{2}(X, m)$. It is useful to record a lemma on suitable cores.

Lemma 6.1. There is an $\mathcal{E}_{1}$-dense subspace $\mathcal{C}$ of $\mathcal{F}$ such that for all $g \in \mathcal{C}$ we have $A g \in \mathcal{B}$. Its image $\partial(\mathcal{C})$ under the derivation $\partial$ is contained in dom $\partial^{*}$.

Proof. Set $\mathcal{C}:=\left\{G_{1} f: f \in \mathcal{F} \cap C(X)\right\}$. Each $g \in \mathcal{F}$ is of the form $g=(A-I)^{-1 / 2} h$ with $h \in L_{2}(X, m)$. As the range of $G_{1}$ is dense in $L_{2}(X, m)$, the function $h$ can be approximated in the $L_{2}(X, m)$-norm by a sequence of functions $G_{1} h_{n}$ with $h_{n} \in \mathcal{F} \cap C_{0}(X)$, and therefore $g$ can be approximated in $\mathcal{E}_{1}$ by the functions $G_{1} h_{n}$. For $g=G_{1} f \in \mathcal{C}$ with $f \in \mathcal{F} \cap C(X)$ we have $A g=f+G_{1} f \in \mathcal{F}$, and the Markov property implies

$$
\left\|G_{1} f\right\|_{L_{\infty}(X, m)} \leq \int_{0}^{\infty} e^{-t}\left\|P_{t} f\right\|_{L_{\infty}(X, m)} d t \leq\|f\|_{L_{\infty}(X, m)} .
$$

The last statement follows from (32).

After these preliminaries we can define the Hodge Laplacian. Set

$$
\operatorname{dom} \Delta_{1}:=\left\{\omega \in \operatorname{dom} \partial^{*}: \partial^{*} \omega \in \mathcal{F}\right\} \text {. }
$$

Definition 6.1. The operator $\Delta_{1}$ with domain dom $\Delta_{1}$ on $\mathcal{H}$, given by

$$
\Delta_{1} \omega:=\partial \partial^{*} \omega, \omega \in \operatorname{dom} \Delta_{1},
$$

will be called the Hodge Laplacian on 1-forms associated with $(\mathcal{E}, \mathcal{F})$.

Theorem 6.1. The Hodge Laplacian $\left(\Delta_{1}\right.$, dom $\left.\Delta_{1}\right)$ is a self-adjoint operator on $\mathcal{H}$.

Note that by (22) and (31) we have

$$
\operatorname{ker} \partial^{*}=\mathcal{H}^{1} .
$$


Proof. Let $\mathcal{C}$ be an $\mathcal{E}$-dense subspace of $\mathcal{F}$ such that $\partial(\mathcal{C})$ is dense in $\operatorname{Im} \partial$ and for all $f \in \mathcal{C}$, we have $A f \in \mathcal{F}$. By Lemma 6.1 such a space always exists. For $\partial f$ with $f \in \mathcal{C}$ and $A f=g \in \mathcal{F}$ we have

$$
\Delta_{1}(\partial f)=\partial(A f)=\partial g
$$

by (32) and (33). Therefore $\partial(\mathcal{C}) \subset \operatorname{dom} \Delta_{1}$, and combining this with (34), dom $\Delta_{1}$ is seen to be dense in $\mathcal{H}$. For any $\omega \in$ dom $\Delta_{1}$ we have the identity

$$
\left\langle\Delta_{1} \eta, \omega\right\rangle_{\mathcal{H}}=-\left\langle\partial^{*} \eta, \partial^{*} \omega\right\rangle_{L_{2}(K, \nu)}=\left\langle\eta, \Delta_{1} \omega\right\rangle_{\mathcal{H}}, \quad \eta \in \operatorname{dom} \Delta_{1},
$$

because $\Delta_{1} \omega=\partial \partial^{*} \omega \in L_{2}(X, m)$. This shows that dom $\Delta_{1} \subset \operatorname{dom} \Delta_{1}^{*}$ and $\Delta_{1}^{*} \omega=\Delta_{1} \omega$ for all $\omega \in \operatorname{dom} \Delta_{1}$.

Remark 6.1. Our Definition 6.1 is adequate if the space of 2 -forms is trivial. In the introduction we have already pointed out that several of our preceding results suggest that this is the case.

Based on Definition 6.1 harmonic 1-forms can be defined.

Definition 6.2. A 1 -form $\omega \in \mathcal{H}$ is called harmonic if $\omega \in \operatorname{dom} \Delta_{1}$ and $\Delta_{1} \omega=0$.

Obviously they form a subspace of $\mathcal{H}$. Moreover, we have the following result.

Theorem 6.2. A 1-form $\omega \in \mathcal{H}$ is harmonic if and only if it is a member of $\mathcal{H}^{1}(X)$.

We will refer to $\mathcal{H}^{1}(X)$ as the space of harmonic 1-forms.

Proof. If $\omega \in \mathcal{H}^{1}(X)=\operatorname{ker} \partial^{*}$, then it is obviously in dom $\Delta_{1}$ and $\Delta_{1} \omega=0$. Conversely, if $\omega \in \mathcal{H}$ is harmonic, then $\Delta_{1} \omega \in$ ker $\partial^{*}$ since

$$
\left\langle\partial u, \Delta_{1} \omega\right\rangle_{\mathcal{H}}=0=\left\langle u, \omega^{*}\right\rangle
$$

holds for all $u \in \mathcal{F}$ if $\omega^{*}=0$. Consequently $\partial^{*} \omega=0$, that is, $\omega \in$ ker $\partial^{*}$.

Finally, recall the definition (19) of the bilinear map $\Gamma_{\mathcal{H}}$. It is the derivation $\partial \Gamma_{\mathcal{H}}(u)$ of $\Gamma_{\mathcal{H}}(u), u \in \mathcal{H}$, that will be needed below. We define it as a linear functional on the possibly smaller domain

$$
\operatorname{dom}_{c} \partial^{*}:=\left\{v \in \operatorname{dom} \partial^{*}: \partial^{*} v \in C(X)\right\} \subset \operatorname{dom} \partial^{*}
$$

by setting

$$
\partial \Gamma_{\mathcal{H}}(u)(v):=-\Gamma_{\mathcal{H}}(u)\left(\partial^{*} v\right)=-\int_{X} \partial^{*} v d \Gamma_{\mathcal{H}}(u), \quad v \in \operatorname{dom}_{c} \partial^{*} .
$$

Note that for any divergence free vector field $v \in \mathcal{H}^{1}(X)$ we have $\partial \Gamma_{\mathcal{H}}(u)(v)=0$. Similarly, given an arbitrary Borel measure $\mu$ on $X$, let a functional $\partial \mu$ on $\operatorname{dom}_{c} \partial^{*}$ be defined by

$$
\partial \mu(v):=-\int_{X} \partial^{*} v d \mu, \quad v \in \operatorname{dom}_{c} \partial^{*}
$$




\section{NAVIER-Stokes EQUATIONS ON FRACTALS}

We finally come to an application of the results developed in Sections 4 and 5 , More precisely, we will consider a Navier-Stokes type system of PDE on a compact connected topologically one-dimensional space $X$ and use Theorem 4.2 together with the decomposition (22) to show that just as in classical smooth cases, only steady state solutions exist for the boundary free case. In this case nontrivial solutions can exist if and only if $\check{H}^{1}(X)$ is nontrivial. If a boundary is specified, further nontrivial solutions may exist. For resistance forms this will be discussed in the next section. Note that these results depend only on the topological dimension; cf. Remark 7.1 below.

For an incompressible and homogeneous fluid in a Euclidean domain the NavierStokes system with viscosity $\nu>0$ and zero outer forcing writes

$$
\left\{\begin{array}{l}
\frac{\partial u}{\partial t}+(u \cdot \nabla) u-\nu \Delta u+\nabla p=0, \\
\operatorname{div} u=0,
\end{array}\right.
$$

where $u$ is the velocity field, $p$ the pressure, and a solution consists of both. See for instance [1, 16, 65]. On a smooth one-dimensional manifold the system reduces to the Euler type equation

$$
\frac{\partial u}{\partial t}+\frac{\partial p}{\partial x}=0
$$

Taking into account the classical polar decomposition $u=\nabla \varphi+w$ of vector fields into a gradient and a solenoidal part and denoting by $\mathcal{P}$ the projection onto the space of solenoidal fields, $u$ is seen to be a stationary solution (steady state solutions) because

$$
0=-\mathcal{P}\left(\frac{\partial p}{\partial x}\right)=\mathcal{P}\left(\frac{\partial u}{\partial t}\right)=\frac{\partial u}{\partial t} .
$$

The aim of this section is to record that a similar behavior occurs for some counterpart of (37) within our setup and under the assumption that the topological dimension of $X$ is less than two.

Remark 7.1. We emphasize that $X$ does not have to possess any smoothness properties, and therefore the sequel applies in particular to highly singular spaces like finitely ramified fractals [48,68, generalized Sierpinski carpets [4, 5, 57] or Barlow-Evans-Laakso spaces [7, 42,61,63, with examples of any possible Hausdorffdimension $1 \leq d<\infty$ among them.

Recall that $X$ is assumed to be a compact connected topologically one-dimensional metric space. We rewrite the divergence condition $\partial^{*} u=0$. By Theorem 4.2. (22) and (31) we have $\operatorname{ker} \partial^{*}=\mathcal{H}^{1}(X)$, therefore prospective solutions will be elements of $\mathcal{H}^{1}(X)$. We use the Hodge Laplacian $\Delta_{1}$ according to (33) and replace the convection term by $\frac{1}{2} \partial \Gamma_{\mathcal{H}}(u)$. With these substitutions (37) can formally be restated as

$$
\left\{\begin{array}{l}
\frac{\partial u}{\partial t}+\frac{1}{2} \partial \Gamma_{\mathcal{H}}(u)-\nu \Delta_{1} u+\partial p=0 \\
\partial^{*} u=0
\end{array}\right.
$$

This is the Navier-Stokes system on $X$ that we investigate.

Remark 7.2. Of course this is just one model, and already in the smooth case there is ambiguity as to which model to formulate (see for instance [58] and the references 
cited therein), let alone in our case. Our motivations to use formulation (38) are as follows.

For a vector field $\left(u=\left(u_{1}, u_{2}, u_{3}\right)\right.$ on $\mathbb{R}^{3}$ we have the identity

$$
\frac{1}{2} \nabla|u|^{2}=(u \cdot \nabla) u+u \times \operatorname{curl} u .
$$

In classical vector analysis on $\mathbb{R}^{3}$ usually 1 - and 2 -forms are identified with vector fields and 0 - and 3-forms with functions. In terms of differential forms curl $u$ is defined (respectively recovered) by viewing the vector field $u$ as a 1 -form, taking its derivation, which is a 2-form, considering the image of this 2-form under the Hodge star operator, which again gives a 1-form, and then translating this 1-form back into a vector field curl $u$. See for instance [1]. In the introduction we have already argued that in our setup the space of 2 -forms should be trivial, and also our Hodge Laplacian is defined with this idea in mind; cf. Remark 6.1. If we pursue this idea, curl $u$ should be zero, and a weak version of the remaining identity above should lead to an analog of

$$
-\frac{1}{2} \int|u|^{2} \operatorname{div} v d x=\frac{1}{2} \int v \nabla|u|^{2} d x=\int v(u \cdot \nabla) u d x .
$$

In our case the Euclidean norm should be replaced by a family of norms on the fibers of $\mathcal{H}$ over $X$ as considered in [33, Section 2]. This suggests replacing the expression on the left hand side of (39) by

$$
-\frac{1}{2} \int_{X} \partial^{*} v d \Gamma_{\mathcal{H}}(u)
$$

Note also that for locally exact vector fields the integrand of the middle integral in (39) locally rewrites $\frac{1}{2} v \nabla|\nabla h|^{2}$. In our language this becomes $\frac{1}{2} \partial \Gamma_{\mathcal{H}}(\partial h)(v)$. We finally remark that by polarization $\partial \Gamma_{\mathcal{H}}$ may be seen as a trilinear form on $\mathcal{H}^{1}(X) \times \operatorname{dom}_{c} A \times \operatorname{dom}_{c} A$, where $\operatorname{dom}_{c} A=\{f \in \operatorname{dom} A: A f \in C(X)\}$, such that if $u$ is divergence free, we have $\partial \Gamma_{\mathcal{H}}(u, \partial g)(\partial g)=-\partial \Gamma_{\mathcal{H}}(u, \partial g)(f)$ for any $f, g \in \operatorname{dom}_{c} A$ by the Leibniz rule (16). This reminds us a bit of the classical theory; see [65, Sections I.2.3 and I.2.4].

In the boundary free case a weak formulation of problem (38) can be made rigorous. As usual we will test against divergence free vector fields $v \in \mathcal{H}^{1}=k e r \partial^{*}$. We interpret $\partial p$ in the measure sense (36) and therefore obtain $\partial p(v)=0$ for all such $v$. Now recall the definitions (33) and (35). We say that a square integrable dom $\partial^{*}$-valued function $u$ on $[0, \infty)$ provides a weak solution to (38) with initial condition $u_{0} \in \mathcal{H}^{1}(X)=\operatorname{ker} \partial^{*}$ if

$$
\left\{\begin{array}{l}
\langle u(t), v\rangle_{\mathcal{H}}-\left\langle u_{0}, v\right\rangle_{\mathcal{H}}+\int_{0}^{t} \partial \Gamma_{\mathcal{H}}(u(s))(v) d s+\nu \int_{0}^{t}\left\langle\partial^{*} u(s), \partial^{*} v\right\rangle_{L_{2}(X, m)} d s=0, \\
\partial^{*} u(t)=0
\end{array}\right.
$$

for a.e. $t \in[0, \infty)$ and all $v \in \mathcal{H}^{1}(X)$. By one-dimensionality this system immediately simplifies further to

$$
\left\{\begin{array}{l}
\left\langle u(t)-u_{0}, v\right\rangle_{\mathcal{H}}=0, \\
\partial^{*} u(t)=0
\end{array}\right.
$$

for a.e. $t \in[0, \infty)$ and all such $v$, and we obtain the following result. 
Theorem 7.1. Any weak solution $u$ of (38) is harmonic and stationary, i.e. $u$ is independent of $t \in[0, \infty)$. Given an initial condition $u_{0}$ the corresponding weak solution is uniquely determined.

In the boundary free case the following is a consequence of Theorems 5.1 and 5.2 .

Corollary 7.1. Assume that there exists a cofinal set $\mathbb{V}$ of finite open covers $\mathcal{V}=$ $\left\{V_{\beta}\right\}_{\beta \in I}$ satisfying (26) and such that any disconnecting set for $\mathbb{V}$ has positive capacity. Then a nontrivial solution to (38) exists if and only if $\check{H}^{1}(X)$ is nontrivial.

We conclude the section with a heuristic remark concerning the pressure $p$.

Remark 7.3. We did not define strong solutions to (37), i.e. solutions that do not need testing. However, if there were a differentiable $\operatorname{dom} \partial^{*}$-valued function $u$ such that (37) holds in a measure-valued sense, then this $u$ should also satisfy the weak formulation (38). Therefore any such $u$ must be stationary, and by (35) and (36) we would obtain

$$
p=-\frac{1}{2} \Gamma_{\mathcal{H}}(u)
$$

seen as an equality of measures.

\section{The CASE OF RESISTANCE FORMS}

In this section we additionally assume that the local Dirichlet form under consideration is induced by a regular resistance form $(\mathcal{E}, \overline{\mathcal{F}})$ on $X$. See [40, 50, 51, 68, for background and precise definitions. In the resistance form context Neumann derivatives are well defined, and it is not difficult to see that if the Navier-Stokes system (38) is considered with a nonempty boundary, it may have additional nontrivial solutions arising from solutions of a related Neumann problem.

Let $(\mathcal{E}, \overline{\mathcal{F}})$ be a local resistance form on $X,[50$, Definition 2.8], and let $R$ be the associated resistance metric. We consider the topological space $(X, R)$. An open ball of radius $r>0$ and with center $x \in X$ in this space is denoted by $B_{R}(x, r)$. For any Borel regular measure $m$ on $(X, R)$ such that $0<m(B(x, r))<\infty$, the space $\left(\bar{F} \cap L_{2}(X, m), \mathcal{E}_{1}\right)$ is Hilbert, and denoting by $\mathcal{F}$ the closure of $C_{0}(X) \cap \overline{\mathcal{F}}$ in it, we obtain a local regular Dirichlet form $(\mathcal{E}, \mathcal{F})$ on $L_{2}(X, m)$. See for instance [51, Section 9]. We assume the following:

Assumption 8.1. The space $(X, R)$ is compact, connected, and topologically onedimensional.

Under Assumption 8.1 the results of the previous sections may be applied to the induced Dirichlet form $(\mathcal{E}, \mathcal{F})$.

Remark 8.1. It is conjectured that any set that carries a regular resistance form becomes a topologically one-dimensional space when equipped with the associated resistance metric.

We provide a few notions and references related to resistance forms. Let $B \subset X$ be a finite set. The points of $B$ will be interpreted as boundary points. By $G_{B}$ we denote the Green operator associated with the boundary $B$ with respect to $(\mathcal{E}, \overline{\mathcal{F}})$, [50, Definition 5.6], and $\mathcal{D}_{B, 0}^{L}$ its image in $\overline{\mathcal{F}}$. Let $\mathcal{H}_{B}$ denote the $B$-harmonic 
functions with respect to $(\mathcal{E}, \overline{\mathcal{F}}),\left[50\right.$, Definition 2.16], and note that $\overline{\mathcal{F}}=\bar{F}_{B} \oplus \mathcal{H}_{B}$, where

$$
\overline{\mathcal{F}}_{B}:=\left\{u \in \overline{\mathcal{F}}:\left.u\right|_{B}=0\right\} .
$$

A $B$-harmonic function $h$ is harmonic on $B^{c}$ in the Dirichlet form sense; more precisely, it satisfies

$$
\mathcal{E}(h, \psi)=0
$$

for all $\psi \in \overline{\mathcal{F}}_{B}$. The space $\mathcal{D}^{L}:=\mathcal{D}_{B, 0}^{L}+\mathcal{H}_{B}$ is seen to be independent of the choice of $B$, [50, Theorem 5.10]. For any $u \in \mathcal{D}^{L}$ and any $p \in X$ the Neumann derivative $(d u)_{p}$ of $u$ at $p$ can be defined. We refer the reader to [50, Theorems 6.6 and 6.8]. Now let $\varphi$ be a function on $B$. A function $h_{\varphi} \in \overline{\mathcal{F}}$ is called a solution to the Neumann problem on $B^{c}$ with boundary values $\varphi$ if it is harmonic on $B^{c}$ and satisfies

$$
(d h)_{p}=\varphi(p)
$$

for all $p \in B$. Such a Neumann solution $h_{\varphi}$ exists if and only if $\varphi$ is such that

$$
\sum_{p \in B} \varphi(p)=0
$$

If it exists, it is unique in $\mathcal{H}_{B}$ up to an additive constant. These last two assertions follow from the connectedness of $X$ in the effective resistance metric assumed above. This connectedness is equivalent to the fact that only constant functions have zero energy. Therefore the linear map $\{h(p)\}_{p \in B} \mapsto\left\{(d h)_{p}\right\}_{p \in B}$ is linear with constant kernel, which implies the assertion by elementary linear algebra.

Regarding a nonempty finite set $B \subset X$ as a boundary, the system (38) is to be viewed on $B^{c}$ (playing the role of a domain). Recalling (40), we will therefore use test vector fields $v$ vanishing outside $B^{c}$. Given an open set $U \subset X$, let

$$
\mathcal{H}(U):=\text { closspan }\left\{a \otimes \mathbf{1}_{V}: a \in \mathcal{B}, V \subset U \text { open }\right\} .
$$

For simplicity we will regard $\mathcal{B}_{b}(U)$ as subspace of $\mathcal{B}_{b}(X)$ under continuation by zero. We will use the following result.

\section{Remark 8.2.}

(i) For any open set $U \subset X$ the equality

$$
\mathcal{H}(U)=\text { clos span }\left\{\omega \mathbf{1}_{U}: \omega \in \mathcal{H}\right\}
$$

holds: Clearly $\mathcal{H}(U)$ is contained in the space on the right hand side. To see the converse inclusion, note that the space on the right hand side contains all simple tensors $a \otimes b \in \mathcal{B} \otimes \mathcal{B}_{b}(U)$, which can be seen using a Dynkin type argument as in [30, Theorem 4.1]. Therefore it must also contain all limits of linear combinations of such elements, and by their denseness in $\mathcal{H}$ the desired inclusion follows using (15).

(ii) For a finite set $B \subset X$ the space $\mathcal{H}\left(B^{c}\right)$ is contained in the space $\operatorname{Im} \partial$ : For any $a \otimes \mathbf{1}_{B^{c}} \in \mathcal{B} \otimes \mathcal{B}_{b}\left(B^{c}\right)$ the continuous function $a$ clearly is constant on each of the finitely many distinct points of $B$. Therefore $a \otimes \mathbf{1}_{B^{c}}$ is exact by Proposition 5.1, and by (i) all members of $\mathcal{H}\left(B^{c}\right)$ are such. 
Let $B \subset X$ be finite. We will now say that a square integrable dom $\partial^{*}$-valued function $u$ on $[0, T]$ provides a weak solution to (38) on $B^{c}$ if

$$
\left\{\begin{array}{l}
\langle u(t), v\rangle_{\mathcal{H}}-\langle u(0), v\rangle_{\mathcal{H}}+\int_{0}^{t} \partial \Gamma_{\mathcal{H}}(u(s))(v) d s+\int_{0}^{t}\left\langle\partial^{*} u(s), \partial^{*} v\right\rangle_{L_{2}(X, m)} d s=0, \\
\langle u(t), \partial \psi\rangle_{\mathcal{H}}=0
\end{array}\right.
$$

for a.e. $t \in[0, T]$, all $v \in \operatorname{dom} \partial^{*} \cap \mathcal{H}\left(B^{c}\right)$ and all $\psi \in \overline{\mathcal{F}}_{B}$.

Theorem 8.1. Let Assumption 8.1 be valid and let $B \subset X$ be finite. If $h$ is the unique, up to an additive constant, harmonic function on $B^{c}$ with normal derivatives $\varphi$ on $B$, then

$$
u(t)=\partial h, \quad t \in[0, \infty),
$$

is the unique weak solution to (38) on $B^{c}$ with the Neumann boundary values $\varphi$ on $B$.

Proof. For $u \equiv \partial h$ the right hand side of the first equation vanishes. The divergence condition holds by harmonicity,

$$
\langle\partial h, \partial \psi\rangle_{\mathcal{H}}=\mathcal{E}(h, \psi)=0
$$

for any $\psi \in \overline{\mathcal{F}}_{B}$.

Remark 8.3. Similarly as before, if $h$ were a 'strong' solution to (38) on $B^{c}$, then we should observe the identity

$$
p(t)=-\frac{1}{2} \Gamma(h), \quad t \in[0, \infty) .
$$

\section{ACKNOWLEDGEMENTS}

We are grateful to Michael Röckner for helpful comments concerning the NavierStokes equations.

\section{REFERENCES}

[1] R. Abraham, J. E. Marsden, and T. Ratiu, Manifolds, tensor analysis, and applications, 2nd ed., Applied Mathematical Sciences, vol. 75, Springer-Verlag, New York, 1988. MR960687 (89f:58001)

[2] Martin T. Barlow, Diffusions on fractals, Lectures on probability theory and statistics (SaintFlour, 1995), Lecture Notes in Math., vol. 1690, Springer, Berlin, 1998, pp. 1-121, DOI 10.1007/BFb0092537. MR.1668115 (2000a:60148)

[3] Martin T. Barlow, Which values of the volume growth and escape time exponent are possible for a graph?, Rev. Mat. Iberoamericana 20 (2004), no. 1, 1-31, DOI 10.4171/RMI/378. MR2076770(2005f:60144)

[4] Martin T. Barlow and Richard F. Bass, Brownian motion and harmonic analysis on Sierpinski carpets, Canad. J. Math. 51 (1999), no. 4, 673-744, DOI 10.4153/CJM-1999-031-4. MR.1701339 (2000i:60083)

[5] Martin T. Barlow, Richard F. Bass, Takashi Kumagai, and Alexander Teplyaev, Uniqueness of Brownian motion on Sierpiński carpets, J. Eur. Math. Soc. (JEMS) 12 (2010), no. 3, 655-701. MR 2639315 (2011i:60146)

[6] Martin T. Barlow, Richard F. Bass, and Takashi Kumagai, Stability of parabolic Harnack inequalities on metric measure spaces, J. Math. Soc. Japan 58 (2006), no. 2, 485-519. MR2228569 (2007f:60064)

[7] Martin T. Barlow and Steven N. Evans, Markov processes on vermiculated spaces, Random walks and geometry, Walter de Gruyter GmbH \& Co. KG, Berlin, 2004, pp. 337-348. MR2087787 (2006b:60164) 
[8] Martin T. Barlow, Alexander Grigor'yan, and Takashi Kumagai, On the equivalence of parabolic Harnack inequalities and heat kernel estimates, J. Math. Soc. Japan 64 (2012), no. 4, 1091-1146, DOI 10.2969/jmsj/06441091. MR2998918

[9] Richard F. Bass, A stability theorem for elliptic Harnack inequalities, J. Eur. Math. Soc. (JEMS) 15 (2013), no. 3, 857-876, DOI 10.4171/JEMS/379. MR3085094

[10] Gregory Berkolaiko and Peter Kuchment, Introduction to quantum graphs, Mathematical Surveys and Monographs, vol. 186, American Mathematical Society, Providence, RI, 2013. MR3013208

[11] Raoul Bott and Loring W. Tu, Differential forms in algebraic topology, Graduate Texts in Mathematics, vol. 82, Springer-Verlag, New York, 1982. MR658304 (83i:57016)

[12] Nicolas Bouleau and Francis Hirsch, Dirichlet forms and analysis on Wiener space, de Gruyter Studies in Mathematics, vol. 14, Walter de Gruyter \& Co., Berlin, 1991. MR1133391 (93e:60107)

[13] E. A. Carlen, S. Kusuoka, and D. W. Stroock, Upper bounds for symmetric Markov transition functions (English, with French summary), Ann. Inst. H. Poincaré Probab. Statist. 23 (1987), no. 2, suppl., 245-287. MR898496 (88i:35066)

[14] J. Cheeger, Differentiability of Lipschitz functions on metric measure spaces, Geom. Funct. Anal. 9 (1999), no. 3, 428-517, DOI 10.1007/s000390050094. MR1708448 (2000g:53043)

[15] Zhen-Qing Chen and Masatoshi Fukushima, Symmetric Markov processes, time change, and boundary theory, London Mathematical Society Monographs Series, vol. 35, Princeton University Press, Princeton, NJ, 2012. MR2849840 (2012m:60176)

[16] A. J. Chorin and J. E. Marsden, A mathematical introduction to fluid mechanics, 2nd ed., Texts in Applied Mathematics, vol. 4, Springer-Verlag, New York, 1990. MR1058010 (91e:76002)

[17] Kai Lai Chung, Lectures from Markov processes to Brownian motion, Grundlehren der Mathematischen Wissenschaften [Fundamental Principles of Mathematical Science], vol. 249, Springer-Verlag, New York, 1982. MR648601 (84c:60091)

[18] F. Cipriani, D. Guido, T. Isola, J.-L. Sauvageot, Differential 1-forms, their integrals and potential theory on the Sierpinski gasket, preprint ArXiv (2011).

[19] Fabio Cipriani and Jean-Luc Sauvageot, Derivations as square roots of Dirichlet forms, J. Funct. Anal. 201 (2003), no. 1, 78-120, DOI 10.1016/S0022-1236(03)00085-5. MR1986156 (2004e:46080)

[20] Fabio Cipriani and Jean-Luc Sauvageot, Fredholm modules on P.C.F. self-similar fractals and their conformal geometry, Comm. Math. Phys. 286 (2009), no. 2, 541-558, DOI 10.1007/s00220-008-0673-4. MR2472035 (2010d:58029)

[21] Samuel Eilenberg and Norman Steenrod, Foundations of algebraic topology, Princeton University Press, Princeton, New Jersey, 1952. MR0050886 (14,398b)

[22] Ryszard Engelking, Dimension theory, North-Holland Publishing Co., Amsterdam, 1978. Translated from the Polish and revised by the author; North-Holland Mathematical Library, 19. MR0482697 (58 \#2753b)

[23] Masatoshi Fukushima, Yōichi Ōshima, and Masayoshi Takeda, Dirichlet forms and symmetric Markov processes, de Gruyter Studies in Mathematics, vol. 19, Walter de Gruyter \& Co., Berlin, 1994. MR.1303354 (96f:60126)

[24] Mariano Giaquinta, Giuseppe Modica, and Jiří Souček, Cartesian currents in the calculus of variations. I, Ergebnisse der Mathematik und ihrer Grenzgebiete. 3. Folge. A Series of Modern Surveys in Mathematics [Results in Mathematics and Related Areas. 3rd Series. A Series of Modern Surveys in Mathematics], vol. 37, Springer-Verlag, Berlin, 1998. Cartesian currents. MR.1645086 (2000b:49001a)

[25] B. M. Hambly, Brownian motion on a homogeneous random fractal, Probab. Theory Related Fields 94 (1992), no. 1, 1-38, DOI 10.1007/BF01222507. MR1189083 (93m:60163)

[26] B. M. Hambly, Brownian motion on a random recursive Sierpinski gasket, Ann. Probab. 25 (1997), no. 3, 1059-1102, DOI 10.1214/aop/1024404506. MR/1457612 (98i:60072)

[27] B. M. Hambly, V. Metz, and A. Teplyaev, Self-similar energies on post-critically finite self-similar fractals, J. London Math. Soc. (2) 74 (2006), no. 1, 93-112, DOI 10.1112/S002461070602312X. MR2254554(2007i:31011)

[28] Juha Heinonen, Lectures on analysis on metric spaces, Universitext, Springer-Verlag, New York, 2001. MR:1800917(2002c:30028) 
[29] Juha Heinonen and Pekka Koskela, Quasiconformal maps in metric spaces with controlled geometry, Acta Math. 181 (1998), no. 1, 1-61, DOI 10.1007/BF02392747. MR1654771 (99j:30025)

[30] Michael Hinz, 1-forms and polar decomposition on harmonic spaces, Potential Anal. 38 (2013), no. 1, 261-279, DOI 10.1007/s11118-012-9272-2. MR3010780

[31] M. Hinz, D. Kelleher, and A. Teplyaev, Measures and Dirichlet forms under the Gelfand transform (English, with English and Russian summaries), Zap. Nauchn. Sem. S.-Peterburg. Otdel. Mat. Inst. Steklov. (POMI) 408 (2012), no. Veroyatnost i Statistika. 18, 303-322, 329-330. MR3032223

[32] M. Hinz, D. Kelleher, and A. Teplyaev, Metrics and spectral triples for Dirichlet and resistance forms, preprint

[33] M. Hinz, M. Röckner, and A. Teplyaev, Vector analysis for regular Dirichlet forms and quasilinear PDE and SPDE on fractals, preprint, to appear in Stoch. Proc. Appl. arXiv:1202.0743 (2012).

[34] M. Hinz and A. Teplyaev, Vector analysis on fractals and applications, to appear in Contemporary Math. arXiv:1207.6375

[35] Michael Hinz and Alexander Teplyaev, Dirac and magnetic Schrödinger operators on fractals, J. Funct. Anal. 265 (2013), no. 11, 2830-2854, DOI 10.1016/j.jfa.2013.07.021. MR3096991

[36] Masanori Hino, On singularity of energy measures on self-similar sets, Probab. Theory Related Fields 132 (2005), no. 2, 265-290, DOI 10.1007/s00440-004-0396-1. MR2199293 (2007g:28010)

[37] Masanori Hino and Kenji Nakahara, On singularity of energy measures on self-similar sets. II, Bull. London Math. Soc. 38 (2006), no. 6, 1019-1032, DOI 10.1112/S0024609306019072. MR2285256(2008c:28008)

[38] M. Hino, Measurable Riemannian structures associated with strong local Dirichlet forms, Math. Nachr. 286 (2013), no. 14-15, 1466-1478. MR.3119694

[39] Masanori Hino, Upper estimate of martingale dimension for self-similar fractals, Probab. Theory Related Fields 156 (2013), no. 3-4, 739-793, DOI 10.1007/s00440-012-0442-3. MR3078285

[40] Marius Ionescu, Luke G. Rogers, and Alexander Teplyaev, Derivations and Dirichlet forms on fractals, J. Funct. Anal. 263 (2012), no. 8, 2141-2169, DOI 10.1016/j.jfa.2012.05.021. MR2964679

[41] Jürgen Jost, Riemannian geometry and geometric analysis, 3rd ed., Universitext, SpringerVerlag, Berlin, 2002. MR1871261 (2002i:53001)

[42] T. J. Laakso, Ahlfors $Q$-regular spaces with arbitrary $Q>1$ admitting weak Poincaré inequality, Geom. Funct. Anal. 10 (2000), no. 1, 111-123, DOI 10.1007/s000390050003. MR.1748917 (2001m:30027)

[43] John M. Lee, Introduction to smooth manifolds, Graduate Texts in Mathematics, vol. 218, Springer-Verlag, New York, 2003. MR1930091 (2003k:58001)

[44] Yves Le Jan, Mesures associées à une forme de Dirichlet. Applications (French), Bull. Soc. Math. France 106 (1978), no. 1, 61-112. MR508949 (81c:31014)

[45] Tom Lindstrøm, Brownian motion on nested fractals, Mem. Amer. Math. Soc. 83 (1990), no. 420, iv+128, DOI 10.1090/memo/0420. MR.988082 (90k:60157)

[46] Naotaka Kajino, Heat kernel asymptotics for the measurable Riemannian structure on the Sierpinski gasket, Potential Anal. 36 (2012), no. 1, 67-115, DOI 10.1007/s11118-011-9221-5. MR.2886454

[47] J. Kigami, Harmonic metric and Dirichlet form on the Sierpiński gasket, diffusions on fractals (Sanda/Kyoto, 1990), Pitman Res. Notes Math. Ser., vol. 283, Longman Sci. Tech., Harlow, 1993, pp. 201-218. MR,1354156 (96m:31014)

[48] Jun Kigami, Analysis on fractals, Cambridge Tracts in Mathematics, vol. 143, Cambridge University Press, Cambridge, 2001. MR1840042 (2002c:28015)

[49] Jun Kigami, Measurable Riemannian geometry on the Sierpinski gasket: the Kusuoka measure and the Gaussian heat kernel estimate, Math. Ann. 340 (2008), no. 4, 781-804, DOI 10.1007/s00208-007-0169-0. MR2372738 (2009g:60105)

[50] Jun Kigami, Harmonic analysis for resistance forms, J. Funct. Anal. 204 (2003), no. 2, 399-444, DOI 10.1016/S0022-1236(02)00149-0. MR2017320(2004m:31010) 
[51] Jun Kigami, Resistance forms, quasisymmetric maps and heat kernel estimates, Mem. Amer. Math. Soc. 216 (2012), no. 1015, vi+132, DOI 10.1090/S0065-9266-2011-00632-5. MR2919892

[52] Jun Kigami, Robert S. Strichartz, and Katharine C. Walker, Constructing a Laplacian on the diamond fractal, Experiment. Math. 10 (2001), no. 3, 437-448. MR.1917429 (2003d:28013)

[53] Pekka Koskela and Yuan Zhou, Geometry and analysis of Dirichlet forms, Adv. Math. 231 (2012), no. 5, 2755-2801, DOI 10.1016/j.aim.2012.08.004. MR2970465

[54] Shigeo Kusuoka, Dirichlet forms on fractals and products of random matrices, Publ. Res. Inst. Math. Sci. 25 (1989), no. 4, 659-680, DOI 10.2977/prims/1195173187. MR.1025071 (91m:60142)

[55] S. Kusuoka, Lecture on diffusion process on nested fractals. Lecture Notes in Math. 1567 39-98, Springer-Verlag, Berlin, 1993.

[56] Zhi Ming Ma and Michael Röckner, Introduction to the theory of (nonsymmetric) Dirichlet forms, Universitext, Springer-Verlag, Berlin, 1992. MR 1214375 (94d:60119)

[57] John M. Mackay, Jeremy T. Tyson, and Kevin Wildrick, Modulus and Poincaré inequalities on non-self-similar Sierpiński carpets, Geom. Funct. Anal. 23 (2013), no. 3, 985-1034, DOI 10.1007/s00039-013-0227-6. MR.3061778

[58] Takeyuki Nagasawa, Navier-Stokes flow on Riemannian manifolds, Proceedings of the Second World Congress of Nonlinear Analysts, Part 2 (Athens, 1996), 1997, pp. 825-832, DOI 10.1016/S0362-546X(96)00375-6. MR 1487663 (98m:35164)

[59] Luke G. Rogers and Alexander Teplyaev, Laplacians on the basilica Julia sets, Commun. Pure Appl. Anal. 9 (2010), no. 1, 211-231, DOI 10.3934/cpaa.2010.9.211. MR2556753 (2011c:28024)

[60] Michael Röckner and Tusheng Zhang, Stochastic 3D tamed Navier-Stokes equations: existence, uniqueness and small time large deviation principles, J. Differential Equations 252 (2012), no. 1, 716-744, DOI 10.1016/j.jde.2011.09.030. MR2852224

[61] Benjamin Steinhurst, Diffusions and Laplacians on Laakso, Barlow-Evans, and other fractals, ProQuest LLC, Ann Arbor, MI, 2010. Thesis (Ph.D.)-University of Connecticut. MR 2753167

[62] B. Steinhurst, Uniqueness of locally symmetric Brownian Motion on Laakso Spaces, Potential Anal. 38 (2013), no. 1, 281-298. MR.3010781

[63] B. Steinhurst and A. Teplyaev, Symmetric Dirichlet forms and spectral analysis on BarlowEvans fractals, preprint, 2011.

[64] Robert S. Strichartz, Taylor approximations on Sierpinski gasket type fractals, J. Funct. Anal. 174 (2000), no. 1, 76-127, DOI 10.1006/jfan.2000.3580. MR1761364 (2001i:31018)

[65] Roger Temam, Navier-Stokes equations and nonlinear functional analysis, CBMS-NSF Regional Conference Series in Applied Mathematics, vol. 41, Society for Industrial and Applied Mathematics (SIAM), Philadelphia, PA, 1983. MR764933 (86f:35152)

[66] Alexander Teplyaev, Gradients on fractals, J. Funct. Anal. 174 (2000), no. 1, 128-154, DOI 10.1006/jfan.2000.3581. MR.1761365 (2001h:31012)

[67] Alexander Teplyaev, Energy and Laplacian on the Sierpiński gasket, Fractal geometry and applications: a jubilee of Benoît Mandelbrot. Part 1, Proc. Sympos. Pure Math., vol. 72, Amer. Math. Soc., Providence, RI, 2004, pp. 131-154. MR2112105 (2006i:31011)

[68] Alexander Teplyaev, Harmonic coordinates on fractals with finitely ramified cell structure, Canad. J. Math. 60 (2008), no. 2, 457-480, DOI 10.4153/CJM-2008-022-3. MR2398758 (2008m:60066)

[69] Frank W. Warner, Foundations of differentiable manifolds and Lie groups, Scott, Foresman and Co., Glenview, Ill.-London, 1971. MR0295244 (45 \#4312)

[70] Nik Weaver, Lipschitz algebras and derivations. II. Exterior differentiation, J. Funct. Anal. 178 (2000), no. 1, 64-112, DOI 10.1006/jfan.2000.3637. MR.1800791 (2002g:46040a)

Department of Mathematics, Universität Bielefeld, Postfach 100131, D-33501 BieleFELD, GERMANY

E-mail address: mhinz@math.uni-bielefeld.de

Department of Mathematics, University of Connecticut, Storrs, Connecticut 062693009

E-mail address: teplyaev@math.uconn.edu 